\title{
ANNULAR LINK INVARIANTS FROM THE SARKAR-SEED-SZABÓ SPECTRAL SEQUENCE
}

\author{
LINH TRUONG AND MELISSA ZHANG
}

\begin{abstract}
For a link in a thickened annulus $A \times I$, we define a $\mathbb{Z} \oplus \mathbb{Z} \oplus \mathbb{Z}$ filtration on Sarkar-Seed-Szabó's perturbation of the geometric spectral sequence. The filtered chain homotopy type is an invariant of the isotopy class of the annular link. From this, we define a two-dimensional family of annular link invariants and study their behavior under cobordisms. In the case of annular links obtained from braid closures, we obtain a necessary condition for braid quasipositivity and a sufficient condition for right-veeringness, as well as Bennequin-type inequalities.
\end{abstract}

\section{INTRODUCTION}

In 2000, Khovanov introduced his categorified knot invariant now known as $\mathfrak{s l}(2)$ homology or Khovanov homology [Kho00]. Soon after, Ozsváth-Szabó [OS04 and Rasmussen [Ras03] independently introduced knot Floer homology, which arises from a filtration on Heegaard Floer homology. Among their many topological applications, knot Floer homology and Khovanov homology have led to a wealth of concordance invariants.

Ozsváth and Szabó extracted information on the four-ball genus of a knot by constructing the concordance invariant $\tau$ OS03, defined using a filtration induced by a knot on the Heegaard Floer chain complex of the three-sphere. Through a similar algebraic construction as that for $\tau$, Rasmussen defined a concordance invariant $s$ [Ras10], which is derived from a filtration spectral sequence from Khovanov homology to Lee's perturbation [Lee05] of Khovanov homology.

The concordance invariants $\tau$ and $s$ are defined using a single filtration grading on a Heegaard Floer or Khovanov complex. Concordance invariants constructed using two linearly independent filtration gradings include

- Ozsváth-Stipsicz-Szabó's concordance homomorphism $\Upsilon_{t}$ [SS17, a piecewise linear function that generalizes the Ozsváth-Szabó $\tau$ invariant. The $\Upsilon_{t}$ invariant is defined using the $\mathbb{Z} \oplus \mathbb{Z}$-filtered knot Floer chain complex $\operatorname{CFK}^{\infty}(K)$.

- Grigsby-Licata-Wehrli's $d_{t}$ GLW17, a smooth annular link invariant with applications to annular link cobordisms and braids, defined from the $\mathbb{Z} \oplus \mathbb{Z}$-filtered annular Khovanov-Lee complex.

- the Sarkar-Seed-Szabó generalized Rasmussen invariants $s^{\mathcal{U}}$ [SSS17] derived from a perturbation of the Szabó geometric spectral sequence and the Bar Natan complex.

- Lewark-Lobb's smooth concordance invariants $\mathbf{I}_{n}$ [LL19], piecewise linear functions arising from Khovanov-Rozansky's complex for $\mathfrak{s l}_{n}$ knot cohomology over the ring $\mathbb{C}[x] / x^{n-1}(x-1)$.

- Dai-Hom-Stoffregen-Truong's concordance homomorphisms $\phi_{j}$ [DHST18] defined using the knot Floer chain complex over the ring $\mathcal{R}=\mathbb{E}[U, V] / U V$.

The first author was partially supported by NSF grant DMS-1606451. 
We study the Sarkar-Seed-Szabó perturbation of the geometric spectral sequence in the setting of annular links, that is, isotopy classes of links in a thickened annulus $A \times I$, or equivalently, a solid torus $D^{2} \times S^{1}$. We will show that for an annular link, the SarkarSeed-Szabó complex admits an annular filtration. This extra filtration allows us to define a two-dimensional family of real-valued annular link invariants $s_{r, t}$, by using three filtration gradings on the Sarkar-Seed-Szabó complex. This family of invariants recovers the SarkarSeed-Szabó generalized $s$-invariants and shares many properties with Grigsby-Licata-Wehrli's $d_{t}$ annular link invariants from Khovanov-Lee homology (cf. [GLW17, Theorem 1).

Theorem 1.1. Let $(L, o)$ be an annular link $L \subset A \times I$ equipped with an orientation $o$. Let $r \in[0,1], t \in[0,1]$.

(1) For each pair $r, t \in[0,1], s_{r, t}(L, o)$ is an oriented annular link invariant.

(2) Suppose $L$ is a knot. Then $s_{0,0}(L, o)=s_{\mathbb{F}_{2}}(L, o)-1$, where $s_{\mathbb{F}_{2}}$ is Rasmussen's concordance invariant over $\mathbb{F}_{2}$ coefficients [Tur06, MTV07].

(3) For fixed $r$ (respectively $t$ ), the function $s_{r, t}(L, o)$ is piecewise-linear with respect to the variable $t$ (respectively $r$ ).

(4) Let $\omega$ be the wrapping number of $L$. Fix $r \in[0,1]$. Then for all $t_{0} \in[0,1]$

$$
\left(-\frac{1}{1-r}\right) \lim _{t \rightarrow t_{0}+} \frac{s_{r, t}(L, o)-s_{r, t_{0}}(L, o)}{t-t_{0}} \in\{-\omega,-\omega+2, \ldots, \omega-2, \omega\} .
$$

(5) Let $F:(L, o) \rightarrow\left(L^{\prime}, o^{\prime}\right)$ be an oriented cobordism between two nonempty, oriented links such that each component of $F$ has a boundary component in $L$. Let $a_{0}$ be the number of annular births or deaths, $a_{1}$ the number of saddles, and $b_{0}$ the number of non-annular births or deaths. Then

$$
s_{r, t}(L, o)-s_{r, t}\left(L^{\prime}, o^{\prime}\right) \leq(r-1)\left(a_{0}-a_{1}+b_{0}(1-t)\right) .
$$

If furthermore each component in $F$ has a boundary component in $L^{\prime}$ as well, then

$$
\left|s_{r, t}(L, o)-s_{r, t}\left(L^{\prime}, o^{\prime}\right)\right| \leq(r-1)\left(a_{0}-a_{1}+b_{0}(1-t)\right) .
$$

(6) $s_{r, t}(L, o)$ is an annular concordance invariant.

We apply properties of the annular link invariants in Theorem 1.1 to the study of braid closures. Let $\sigma \in \mathfrak{B}_{n}$ be an $n$-braid. Its braid closure $\widehat{\sigma}$ is naturally an annular link, equipped with a braid-like orientation $o_{\uparrow}$ as described in Section 3.4. We abbreviate $s_{r, t}\left(\widehat{\sigma}, o_{\uparrow}\right)$ by $s_{r, t}(\widehat{\sigma})$. Analogous to properties of the $d_{t}$ invariant [GLW17, Theorem 2, Theorem 4], we obtain a necessary condition for braid quasipositivity and a sufficient condition for rightveeringness. For a fixed $r_{0} \in[0,1]$, we find the function $s_{r_{0}, t}(\widehat{\sigma})$ is piecewise-linear and has slope bounded above by $n$, the braid index of $\sigma$. Let $m_{r_{0}, t_{0}}(L, o)$ denote the right-hand slope, with respect to the variable $t$, of $s_{r_{0}, t}(L, o)$ at $t_{0}$.

Theorem 1.2. If $\sigma$ is a quasipositive braid of index $n$ and writhe $w \geq 0$, we have

$$
s_{r, t}(\widehat{\sigma})=(1-r)(w-(1-t) n)
$$

for all $r \in[0,1]$ and $t \in[0,1]$.

Theorem 1.3. Let $\sigma \in \mathfrak{B}_{n}$. Fix $r_{0}<1$. If $s_{r_{0}, t}(\widehat{\sigma})$ attains maximal slope at some $t=t_{0}<\frac{1}{2}$ (that is, $m_{r_{0}, t_{0}}(\widehat{\sigma})=n$ for some $t_{0} \in\left[0, \frac{1}{2}\right)$ ), then $\sigma$ is right-veering.

In addition, we study the behavior of $s_{r, t}(\widehat{\sigma})$ under Markov stabilizations. We achieve bounds which can be compared to the analogous $d_{t}$ bounds ([GLW17], Proposition 5). 
Proposition 1.4. Let $\sigma \in \mathfrak{B}_{n}$ and suppose $\sigma^{ \pm} \in \mathfrak{B}_{n+1}$ is obtained from $\sigma$ by either a positive or negative Markov stabilization. Then for all $r \in[0,1], t \in[0,1]$,

$$
s_{r, t}(\widehat{\sigma})-(1-r) t \leq s_{r, t}\left(\widehat{\sigma}^{ \pm}\right) \leq s_{r, t}(\widehat{\sigma})+(1-r) t .
$$

A complete understanding of the behavior of the $s_{r, t}$ (and similarly, $d_{t}$ ) annular link invariants under positive and negative stabilization is currently unknown. This question is related to the question of the effectiveness of transverse invariants obtained from Khovanov homology.

Question 1.5. Suppose $\sigma^{+} \in \mathfrak{B}_{n+1}$ is obtained from $\sigma \in \mathfrak{B}_{n}$ by a positive Markov stabilization. Does the equality

$$
s_{r, t}\left(\widehat{\sigma}^{+}\right)=s_{r, t}(\widehat{\sigma})+(1-r) t
$$

hold for all $r \in[0,1], t \in[0,1]$ ?

A positive answer to Question 1.5 would yield a new transverse invariant: $s_{r, t}(\widehat{\sigma})-n(1-r) t$. It is unclear whether such an invariant would be effective, in the sense that it would provide more information than the self-linking number of the transverse link. The analogous questions with respect to the $d_{t}$ invariant are open, as well as the effectiveness of Plamenevskaya's transverse link invariant $\psi$ [Pla06].

Analogous to the annular Khovanov-Lee setting [GLW17, Corollary 4], we produce a lower bound on the band rank $\mathrm{rk}_{n}$ of a braid [Rud83]. Given $\beta \in \mathfrak{B}_{n}$,

$$
\operatorname{rk}_{n}(\beta):=\min \left\{c \in \mathbb{Z}^{\geq 0} \mid \beta=\prod_{j=1}^{c} \omega_{j} \sigma_{i_{j}}^{ \pm}\left(\omega_{j}\right)^{-1} \text { for some } \omega_{j} \in \mathfrak{B}_{n}\right\},
$$

where $\sigma_{i_{j}}$ denotes the elementary Artin generators. Note that band $\operatorname{rank} \mathrm{rk}_{n}$ is a braid conjugacy class invariant. Topologically, band $\operatorname{rank} \operatorname{rk}_{n}(\beta)$ is the minimum number of halftwist bands (running perpendicularly to the strands) needed to construct a Seifert surface for $\widehat{\beta}$ from $n$ disks (the obvious Seifert surface for the identity braid closure in $\mathfrak{B}_{n}$ ).

Proposition 1.6. Let $r \neq 1$. Given an oriented cobordism $F$ from $(L, o)$ to $\left(L^{\prime}, o^{\prime}\right)$ with $a_{0}$ annular even index critical points, $a_{1}$ annular odd index critical points, and $b_{0}$ non-annular even index critical points, we have

$$
\left|\frac{s_{r, t}(\hat{\beta})}{1-r}+n(1-t)\right| \leq r k_{n}(\beta) .
$$

As described in [GLW17, Gri18, work of Rudolph [Rud83, Sec. 3] suggests that a braid conjugacy class invariant (like $s_{r, t}$ ) that yields a lower bound on band rank could potentially lead to an effective ribbon obstruction. This question is explored using the $d_{t}$ invariant in [Gri18; we do not pursue this question using $s_{r, t}$ here.

The Sarkar-Seed-Szabó theory conjecturally relates the Khovanov and Heegaard Floer worlds by combining the Szabó geometric spectral sequence with Bar Natan's homology. Szabó's geometric spectral sequence [Sza15] is conjecturally isomorphic to the Ozsváth-Szabó spectral sequence [OS05] relating the Khovanov homology of (the mirror of) a link $\bar{L}$ to the Heegaard Floer homology $\widehat{H F}(\Sigma(L))$ of the double branched cover. In addition, F. Lin [Lin19] recently constructed a spectral sequence relating a version of Bar Natan's homology of (the mirror of) a link $\bar{L}$ to the involutive monopole Floer homology $\widetilde{H M I}(\Sigma(L))$ of the double 
branched cover $\Sigma(L)$, which is analogous to the hat version of the involutive Heegaard Floer homology $\widehat{H F I}(\Sigma(L))$ of Hendricks-Manolescu [HM17].

We will study a version of the Sarkar-Seed-Szabó theory for annular links. In [Rob13. Roberts constructed an annular analogue to the Ozsváth-Szabó spectral sequence [OS05]. Therefore, the annular version of Szabó's geometric spectral sequence conjecturally converges to (a variant of) the knot Floer homology of the preimage $\widetilde{B}$ of the annular axis $B$ in the double branched cover. While we study a filtered version of the Sarkar-Seed-Szabó complex, the full Sarkar-Seed-Szabó complex is defined over the two-variable polynomial ring $\mathbb{F}_{2}[H, W]$. It may also be interesting to extend the techniques in this work to, for instance, an annular version of the Sarkar-Seed-Szabó complex over $\mathbb{F}_{2}[H]$ and compare the resulting spectral sequence with that of $\mathrm{F}$. Lin Lin19], potentially yielding a spectral sequence abutting to an involutive knot Floer invariant of $\widetilde{B}$ in the double branched cover $\Sigma(L)$.

1.1. Acknowledgements. We thank Eli Grigsby for her advice and insightful comments on a draft. We also thank John Baldwin, Ciprian Manolescu, Gage Martin, Krzysztof Putyra, Adam Saltz, Sucharit Sarkar, and Matt Stoffregen for interesting conversations. This collaboration began at the Georgia International Topology Conference at the University of Georgia in 2017, and continued at Columbia University while the first author was a postdoc and the second author was a visiting PhD student. We thank UGA and Columbia's math departments for their hospitality.

\section{BACKGROUND}

The purpose of this section is to review the homology theories used in this paper and to set notation.

2.1. Links and their diagrams. We are concerned with defining a functorial invariant for links in $S^{3}$ via link diagrams. Following [BHL19], we first define the relevant categories.

Definition 2.1. Let Link denote the category of smooth links in $S^{3}=\mathbb{R}^{3} \cup\{\infty\}$. Objects are smooth isotopy classes of oriented links in $S^{3}$. Morphisms are smooth isotopy classes of oriented, collared link cobordisms in $S^{3} \times[0,1]$ : two surfaces from $L_{0} \subset S^{3} \times\{0\}$ to $L_{1} \subset S^{3} \times\{1\}$ are equivalent if there is a smooth isotopy taking $F$ to $F^{\prime}$ that fixes a collar neighborhood of the boundary $S^{3} \times\{0,1\}$, pointwise.

Link invariants are typically computed from a link diagram.

Definition 2.2. Let Diag denote the diagrammatic link cobordism category, representing links in $S^{3}$. Objects are oriented link diagrams in $S^{2}$, up to planar isotopy and the Reidemeister moves (see Remark 2.3). Morphisms are diagrammatic cobordisms, usually described as movies, up to a set of equivalences.

Movies are scans of surfaces in $S^{3} \times[0,1]$, where $[0,1]$ is the time component. The scan at a fixed time is called a frame, which can either look like a link diagram or something more singular. We can always arrange the link cobordism via isotopy such that there are only finitely many, isolated singular frames. In this situation, the movie is a composition of planar isotopies interspersed with finitely many elementary string interactions (ESIs): Reidemeister moves or oriented diagrammatic 0-, 1-, and 2-handle attachments. We sometimes refer to the handle attachments as births, saddles, and deaths, respectively, because a tiny snippet of the movie surrounding these critical levels looks like the birth or death of a circle, or 
describes a saddle cobordism. We visually depict ESIs by drawing the first and last frames of this tiny snippet.

Two movies are equivalent if they can be related by a finite sequence of the following moves ([GLW18], Appendix):

- a Carter-Saito movie move [CS93], localized to a disk in $\mathbb{R}^{2}$,

- a time-level preserving isotopy of the associated immersed surfaces,

- an interchange of the time-levels of distant (noninteracting) ESIs.

Remark 2.3. Since every link diagram will miss some point on $S^{2}$, we actually draw the diagrams on $\mathbb{R}^{2}$ and think of isotopies in $\mathbb{R}^{2}$. By Reidemeister's theorem, moving a strand across $\infty$ is equivalent to a finite sequence of Reidemeister moves through diagrams on $\mathbb{R}^{2}$.

A functorial link invariant is a functor from Link. Baldwin-Hedden-Lobb describe how to lift a functor from Diag to a functor from Link, and show that these two categories are equivalent BHL19]. In this paper we will focus on functors into a category of filtered complexes.

Definition 2.4. Let Filt be the homotopy category of filtered complexes. The objects are filtered chain complexes, up to chain homotopy equivalence (we denote this relation by $\simeq$ ), and the morphisms are induced by filtered chain morphisms.

2.2. Annular links and cobordisms. Let $A$ be a closed, oriented annulus and let $I=[0,1]$ be the closed, oriented unit interval. Via the identification

$$
A \times I=\{(r, \theta, z) \mid r \in[1,2], \theta \in[0,2 \pi], z \in[0,1]\} \subset\left(S^{3}=\mathbb{R}^{3} \cup \infty\right),
$$

any link $L \subset A \times I$ may be naturally viewed as a link in the complement of a standardly embedded unknot, $U=(z$-axis $\cup \infty) \subset S^{3}$. Such an annular link $L \subset A \times I$ admits a diagram $\mathcal{D}(L) \subset A$, obtained by projecting a generic isotopy class representative of $L$ onto $A \times\{1 / 2\}$.

We shall view $\mathcal{D}(L)$ as a diagram on $S^{2} \backslash\{\mathbb{O}, \mathbb{X}\}$ where $\mathbb{X}$ (resp. $\mathbb{O}$ ) are basepoints on $S^{2}$ corresponding to the inner (resp. outer) boundary circles of $A$. Note that if we forget the data of $\mathbb{X}$, we may view $\mathcal{D}(L)$ as a diagram on $\mathbb{R}^{2}=S^{2} \backslash \mathbb{O}$ of $L$, viewed as a link in $S^{3}$.

In the annular context, we can categorize these moves as annular or non-annular, based on whether they interact with the axis:

- (1-handles) By transversality, all saddle moves can be thought of as annular.

- (0, 2-handles) There are annular and non-annular births/deaths, depending on whether the circles being born/dying is trivial or not, respectively.

- (Reidemeister moves and planar isotopies) If the local disk in which a Reidemeister move occurs does not include the basepoint, then it is called an annular Reidemeister move; otherwise, it is non-annular. The same goes for planar isotopies.

Remark 2.5. The homology theories / TQFTs of interest will still be defined as functors Link $\rightarrow$ Filt; in particular, the distinguished homology classes come from the link diagram on $S^{2}$. However, the basepoints indicating the presence of the embedded unknot will provide an extra filtration.

2.3. Khovanov (co)chains, gradings, and annular filtration. In this subsection we give a brief review of Khovanov homology and the variants appearing in this paper. We refer the reader to [BN02, Kho00] for more details on the construction, and focus mainly on setting the notation to be used in the rest of the paper. 
Let $\mathbf{2}^{n}$ be the $n$-dimensional Boolean cube $\{0,1\}^{n}$. The vertices $u \in \mathbf{2}^{n}$ are graded by $|u|=\sum_{i} u_{i}$, and there is a directed edge $u \rightarrow v$ whenever $u \preceq v$ and $|v|-|u|=1$.

Let $\mathcal{D}$ be an oriented link diagram with $n$ crossings. The Khovanov (co)chain complex $K c(\mathcal{D})$ lies above $\mathbf{2}^{n}$. In this section we'll only describe the (co)chains, and leave the differentials to a more general discussion in Section 2.5.

Each vertex $u \in 2^{n}$ corresponds to a complete resolution $\mathcal{D}_{u}$ of $\mathcal{D}$. Let $S_{u}$ be the collection of planar circles in this resolution. A marked resolution is this resolution $\mathcal{D}_{u}$ where each circle is decorated with either a + or a - . The vector space $K c$ associates to $u$ is generated by all these marked resolutions. Equivalently, let $\mathbb{V}$ be the two-dimensional vector space generated by $x_{-}$and $x_{+} ; K c\left(\mathcal{D}_{u}\right)$ is then $\mathbb{V} \otimes\left|S_{u}\right|$, where the marked resolution corresponds to a pure tensor. Equivalently, the marked resolution corresponds to a monomial in the symbols $\left\{x_{i}\right\}_{i=1}^{\left|S_{u}\right|}$ where $x_{i}$ belongs to the $i$ th circle. Here is the dictionary relating these three ways of describing the distinguished generators:

- circle $i$ is labeled - in the marked resolution $=x_{-}$in position $i$ in the pure tensor $=$ $x_{i}$ appears in the monomial

- circle $i$ is labeled + in the marked resolution $=x_{+}$in position $i$ in the pure tensor $=$ $x_{i}$ does not appear in the monomial

Marked resolutions are useful in diagrammatic computations; Grigsby-Licata-Wehrli use the pure tensors in [GLW17]; Sarkar-Seed-Szabó use monomials in [SSS17].

Let $\vec{x}$ be a pure tensor in $K c(\mathcal{D}(L))$, located above vertex $u \in \mathbf{2}^{n}$. Let $n_{+}$(resp. $n_{-}$) be the number of positive (resp. negative) crossings in the oriented link diagram $\mathcal{D}$.

- The homological grading $\operatorname{gr}_{h}(\vec{x})$ is given by $|u|-n_{-}$.

- The quantum grading $\operatorname{gr}_{q}(\vec{x})$ is given by $|u|+\#\left(x_{+}\right)-\#\left(x_{-}\right)+n_{+}-2 n_{-}$.

In the presence of the basepoints $\mathbb{X}$ and $\mathbb{O}$, we tweak the definition above to reflect the position of the circles with respect to these basepoints. In a given complete resolution $\mathcal{D}_{u}$, the circles $S_{u}$ fall into two categories: those that separate the basepoints, and those that do not. We refer to the former as nontrivial circles, and the latter as trivial circles. To notationally distinguish these circles in the pure tensor setting, in place of $x_{ \pm}$in the previous paragraphs, we write $v_{ \pm}$for the \pm -labeling of a nontrivial circle and $w_{ \pm}$for the \pm -labeling of a trivial circle. (The quantum grading does not depend on the triviality of circles, e.g. $\left.\#\left(x_{-}\right)=\#\left(v_{-}\right)+\#\left(w_{-}\right).\right)$

We can now associate a third grading to the distinguished generators.

- The $k$-grading $\operatorname{gr}_{k}(\vec{x})$ is given by $\#\left(v_{+}\right)-\#\left(v_{-}\right)$.

The Khovanov differential preserves the homological and quantum gradings, producing a bigraded homology theory. In the annular context, the differential is filtered (non-increasing) with respect to the $k$-grading.

2.4. Szabó's geometric spectral sequence. In [Sza15], Szabó introduced his geometric spectral sequence in Khovanov homology. The underlying chains are the same as those described in Section 2.3. The differentials are described in terms of resolution configurations of index $\geq 1$, described below. Resolution configurations describe individual components of the differential by encoding two distinguished generators - one "before" picture and one "after" picture - and what arcs are used to surger to the "before" picture to arrive at the "after" picture. If a resolution configuration displays the ordered pair of distinguished generators (before, after) $=(\vec{x}, \vec{y})$, then $\vec{y}$ appears in the image of $\vec{x}$ under the differential. 


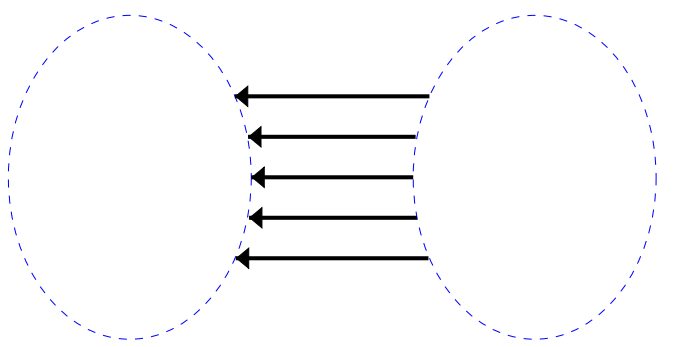

(A) Type A

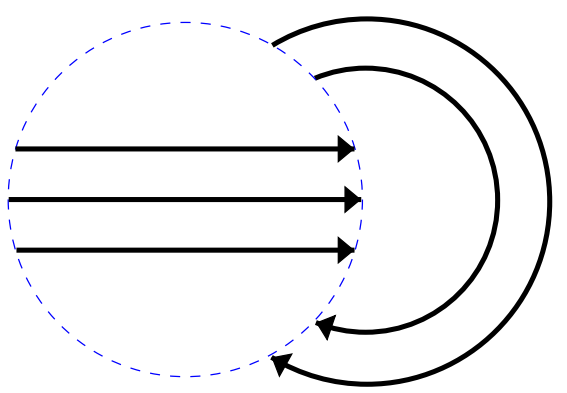

(c) Type $\mathrm{C}$

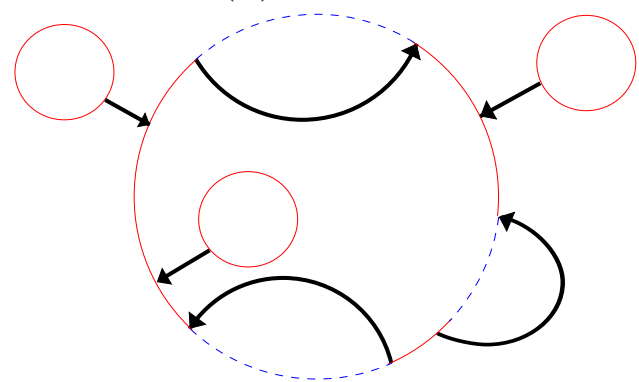

(E) Type E

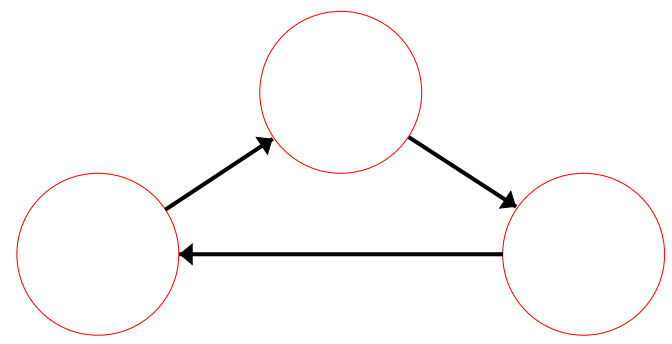

(B) Type B

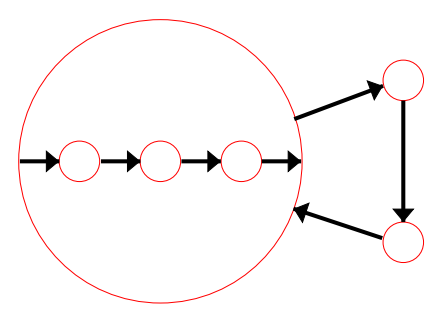

(D) Type D

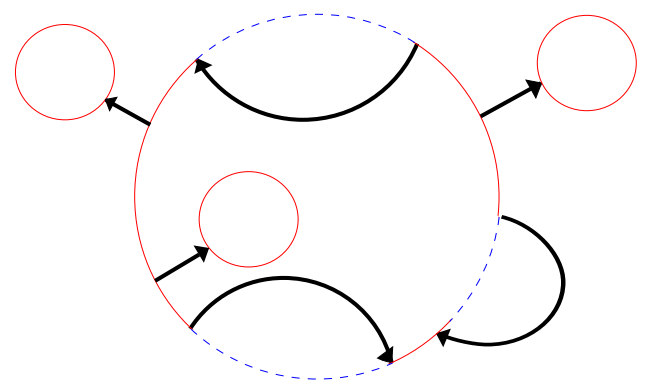

(F) Type E

FigurE 1. The oriented configurations that contribute to the Szabó differential

Figure 1 illustrates the five types of resolution configurations that contribute to the Szabo differential.

Szabó's theory is $\mathrm{gr}_{h}$-filtered (cf. Proposition 3.1). The index 1 resolution configurations correspond to the original Khovanov differential. Thus $\mathrm{gr}_{h}$-filtration gives rise to the "geometric spectral sequence" from Khovanov homology.

2.5. The Sarkar-Seed-Szabó perturbation of the geometric spectral sequence. The Sarkar-Seed-Szabó perturbation adds further differentials to the Szabó complex, inspired by Bar-Natan's deformation of Khovanov homology [BN05]. The index 1 resolution configurations correspond to the original Bar-Natan deformation differentials.

The total chain complex of Sarkar-Seed-Szabó [SSS17] is

$$
\mathcal{C}_{\text {tot }}=\left(\mathcal{C} \otimes \mathbb{E}[H, W], \delta_{\text {tot }}=d_{1}+W d_{2}+W^{2} d_{3}+\cdots+H h_{1}+H W h_{2}+H W^{2} h_{3}+\cdots\right)
$$

where the chain groups $\mathcal{C}$ are the same as the Khovanov chain groups and the total differential lies in (homological, quantum)-grading $(1,0)$. The maps $d_{i}$ correspond to the Szabó differential maps, and we refer the reader to [SSS17] for the definition of the maps $h_{i}$. The 
Bar-Natan deformation of the Khovanov chain complex can be obtained from $\mathcal{C}_{\text {tot }}$ by setting $\mathrm{W}=0$, and the Szabó chain complex can be obtained from $\mathcal{C}_{\text {tot }}$ by setting $H=0$. In this paper, we are mainly interested in the filtered version $\left(\mathcal{C}_{f t o t}, \delta_{f t o t}\right)$ :

$$
\mathcal{C}_{\text {ftot }}=\mathcal{C}_{\text {tot }} /\{H=W=1\}=\left(\mathcal{C}, d_{1}+d_{2}+d_{3}+\cdots+h_{1}+h_{2}+h_{3}+\cdots\right) .
$$

\section{Annular Sarkar-SeEd-Szabó invariants}

3.1. The annular filtration. From the data of the diagram $\mathcal{D}(L) \subset S^{2} \backslash\{\mathbb{O}, \mathbb{X}\}$ of an oriented annular link $L \subset A \times I$, we will use the construction of Sarkar-Seed-Szabó [SSS17], building on constructions of Khovanov [Kho00], Bar-Natan [BN05], and Szabó [Sza15], to define a $\mathbb{Z} \oplus \mathbb{Z} \oplus \mathbb{Z}$-filtered chain complex as follows.

We obtain a third grading (the $k$ grading) on the Sarkar-Seed-Szabó chain complex $\mathcal{C}_{\text {tot }}$, as follows. In the language of [SSS17], a "0" (respectively, "1") marking on a starting circle denotes that the circle appears (respectively, does not appear) in the starting monomial. In a break with their conventions, we write + and - instead of the "0" and "1" that SarkarSeed-Szabó use on starting circles. (This change is made to reserve the use of numbers for the $k$ grading). We keep the [SSS17] notation for the ending monomial: A dashed blue (respectively, solid red) ending circle in a complete resolution of $\mathcal{D}(L)$ denotes that the ending circle does not appear (respectively, does appear) in the ending monomial. Recall that a circle are either trivial (respectively, nontrivial) if it intersects any fixed oriented arc $\gamma$ from $\mathbb{X}$ to $\mathbb{O}$ in an even (respectively, odd) number of points. We define the $k$ grading of a basis element of $\mathcal{C}$ (or a square-free monomial in circles in a complete resolution) to be the number of nontrivial circles not appearing in the monomial minus the number of nontrivial circles appearing in the monomial.

Lemma 3.1. The (filtered) total differential $\delta_{f t o t}$ decomposes into $\left(\mathrm{gr}_{h}, \mathrm{gr}_{q}, \mathrm{gr}_{k}\right)$-homogeneous pieces. In particular,

- $d_{i}$ increases $\operatorname{gr}_{h}$ and $\operatorname{gr}_{q}$ by $i$ and $2 i-2$, respectively, and decomposes into three $\mathrm{gr}_{k}$-homogeneous summands $d_{i,-2}, d_{i, 0}$, and $d_{i, 2}$, corresponding to a shift in $\mathrm{gr}_{k}$ by an element of $-2,0$, and 2 , respectively. Furthermore, $d_{1,2}=0$.

- $h_{i}$ increases $\operatorname{gr}_{h}$ and $\operatorname{gr}_{q}$ by $i$ and $2 i$, respectively, and decomposes into $\operatorname{gr}_{k}$-homogeneous summands $h_{i, j}$ corresponding to a shift in $\operatorname{gr}_{k}$ by an even integer $j \in[0, i+1]$.

Therefore we may write $\delta_{f t o t}=\sum_{i}\left(d_{i}+h_{i}\right)$, where the $d_{i}$ and $h_{i}$ decompose into summands

- $d_{i}=d_{i,-2}+d_{i, 0}+d_{i, 2}$, with $d_{1,2}=0$

- $h_{i}=h_{i, 0}+h_{i, 2}+h_{i, 4}+\cdots+h_{i, i+1}$

and each summand is $\left(\mathrm{gr}_{h}, \mathrm{gr}_{q}, \mathrm{gr}_{k}\right)$-homogeneous.

Proof. The $\mathrm{gr}_{h^{-}}$and $\mathrm{gr}_{q}$-homogeneity of $d_{i}$ and $h_{i}$ are given in [SSS17]. We will check the $k$-grading $\left(\mathrm{gr}_{k}\right)$ shifts of $d_{i}$ and $h_{i}$. First, note that $d_{1}$ coincides precisely with the Khovanov differential. In GLW17, Lemma 3] it is shown that $d_{1}$ splits into homogeneous summands that shift the $k$-grading by either 0 or -2 . Thus, we have $d_{1,2}=0$ as claimed.

We next check the $k$-grading $\operatorname{gr}_{k}$ shift by $d_{i}$. We will consider all possible configurations of the Szabó differential (see Figure 1). For each possible configuration and possible placement of the basepoints $\mathbb{X}$ and $\mathbb{O}$, we compute the $k$-grading difference of the ending and starting monomial associated to the configuration. If $\mathbb{X}$ is located in the same region of the plane as $\mathbb{O}$, then the $k$-grading shift is zero. In the following casework, we leave out this possibility. 


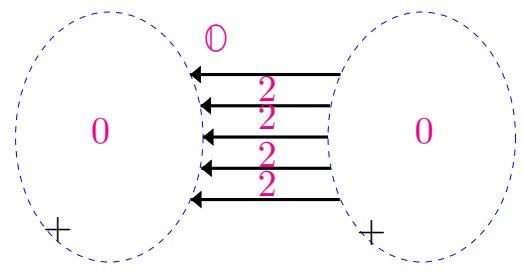

(A) Type A configuration

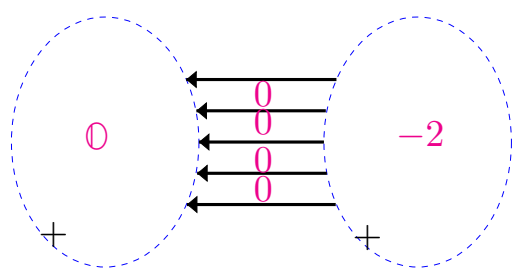

(B) Type A configuration

FiguRE 2. Type A configurations with $k$-grading shifts corresponding to the placement of $\mathbb{X}$ basepoint

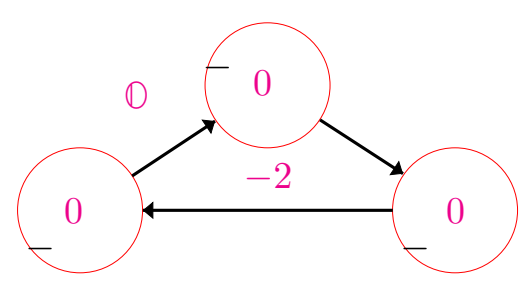

(A) Type B configuration

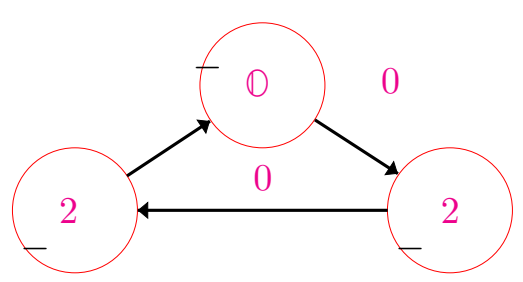

(B) Type B configuration

Figure 3. Type B configurations with $k$-grading shifts

(1) In a Type-A configuration (Figure 1a), there are two starting circles, with some (at least one) parallel arcs between them. Note that after removing the two starting circles from $S^{2}$, there are three connected components: two disks and an annulus. Up to symmetry, the basepoint $\mathbb{O}$ is either inside a disk region bounded by a starting circle (Figure 2b) or inside the unique annulus bounded by the two starting circles (Figure 2a). In every possible type A configuration, the $k$-grading shift is either -2 , 0 , or 2. See Figure 2,

(2) A Type-B configuration (Figure $1 \mathrm{~b}$ ) is the dual of a Type-A configuration. Up to symmetry, there are two possibilities for the placement of $\mathbb{O}$ : either outside the starting circles (Figure 3a) or inside a starting circle (Figure 3b). In every possible type B configuration, the $k$-grading shift is either -2 , 0 , or 2 . See Figure 3 .

(3) In a Type-C configuration (Figure 1c), up to symmetry, there are two possibilities for the placement of $\mathbb{O}$ (Figure $4 \mathrm{c}$ ): the basepoint $\mathbb{O}$ is either between two parallel arcs or not. For each possible type $\mathrm{C}$ configuration, the $k$-grading shift is either 0 or 2 , depending on the location of $\mathbb{X}$. See Figure 4 .

(4) A Type-D configuration (Figure $1 \mathrm{~d}$ ) is the mirror of the dual of a Type-C configuration. Thus, the $k$-grading shift candidates are the same as those of a type $\mathrm{C}$ configuration: 0 or 2 .

(5) In a Type-E configuration (Figure 1e or Figure 1f), up to symmetry there are three possibilities for the location of the $\mathbb{O}$ basepoint: see Figure 5 a, For each possible $\mathbb{X}$ and $\mathbb{O}$ pairing, the $k$-grading shift is either $-2,0$, or 2 . See Figure 5 .

To see that $\mathrm{gr}_{k}$ only ever changes in even number increments, consider the mod 2 nesting of circles. Observe that, with respect to an arc $\gamma$ drawn from $\mathbb{O}$ to $\mathbb{K}$, a surgery does not change the mod 2 intersection number between $\gamma$ and the circles.

To see the $k$-grading shifts of $h_{i}$, recall that the resolution configurations $(R, x, y)$ contributing to $h_{i}$ are disjoint unions of trees and dual trees (see [SSS17, Definition 3.2]). We 


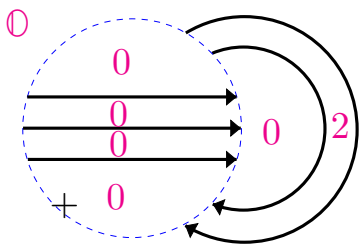

(A) Type C configuration

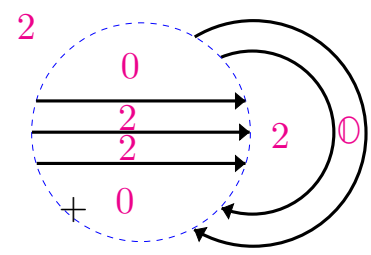

(B) Type C configuration

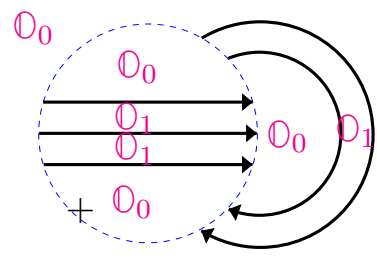

(C) Type C configuration: $\mathbb{O}$ basepoint symmetries

Figure 4. Type C configurations with $k$-grading shifts

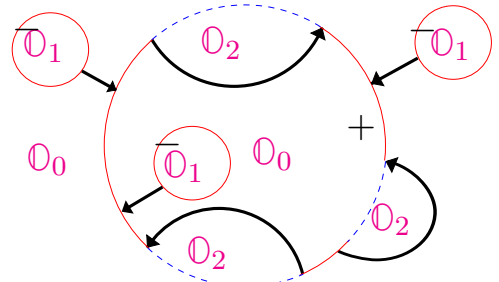

(A) Type E: $\mathbb{O}$ basepoint symmetries

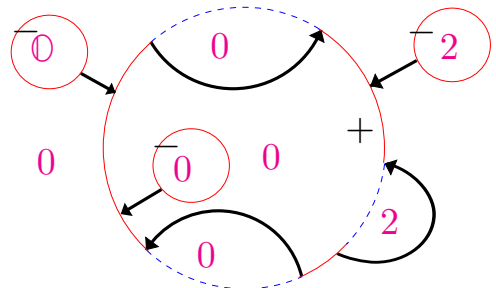

(c) Type E configuration

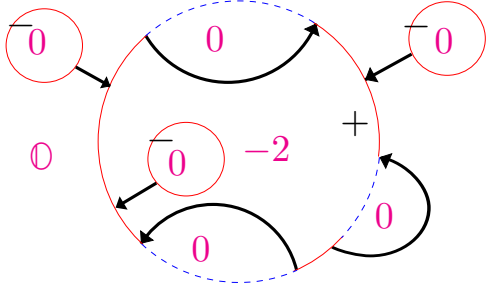

(B) Type E configuration

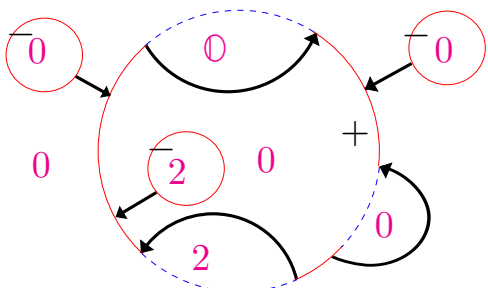

(D) Type E configuration

Figure 5. Type E configurations with $k$-grading shifts

first focus on computing the $k$-grading shift of a single tree. Recall that a tree is a labeled resolution configuration of some index $i \geq 0$, with exactly $(i+1)$ starting circles, exactly one ending circle, and no passive circles (unless $i=0$ ), and with all the starting circles appearing in the starting monomial, and the ending circle appearing in the ending monomial. Therefore, the initial $k$-grading must be a non-positive integer in the interval $[-(i+1), 0]$, while the ending $k$-grading is either 0 or -1 . Moreover, it is easy to check that $k$-grading increases by twice the number of nontrivial node circles in the tree. Therefore, the $\mathrm{gr}_{k}$ shift must be an even integer. Thus, an index $i$ tree configuration contributes to $h_{i}$ (increasing the homological grading $\operatorname{gr}_{h}$ by $i$ ) and must increase the $k$-grading by an even integer in $[0, i+1]$. 
Next, observe that a configuration and its dual admit the same $k$-grading shifts. Therefore, the $k$-grading shift of a single dual tree of index $i$ is also an even integer in $[0, i+1]$.

Finally we analyze the behavior of the $k$-grading shift under disjoint unions of trees and dual trees. Given any resolution configuration $(R, x, y)$, we may find an "equator" circle $C$ on the sphere which does not intersect any of the arcs or circles in the resolution configuration. This "equator" circle $C$ does not belong to the circles in $(R, x, y)$. Moreover, $C$ divides the sphere into two disks $D_{1}$ and $D_{2}$ containing the two resolution configurations $\left(R_{1}, x_{1}, y_{1}\right)$ and $\left(R_{2}, x_{2}, y_{2}\right)$ respectively. We have $(R, x, y)=\left(R_{1} \sqcup R_{2}, x_{1} x_{2}, y_{1} y_{2}\right)$.

(1) If the basepoints $\mathbb{O}$ and $\mathbb{X}$ lie in the same disk, say $D_{1}$, then the $k$-grading shift of the resolution configuration $(R, x, y)$ is the same as the $k$-grading shift of a resolution configuration $\left(R_{1}, x_{1}, y_{1}\right)$.

(2) If the basepoints $\mathbb{O}$ and $\mathbb{X}$ lie in the disks $D_{1}$ and $D_{2}$ respectively, then the overall $k$ grading shift of the resolution configuration $(R, x, y)$ equals the sum of the following two quantities:

- the $k$-grading shift $\left(R_{1}, x_{1}, y_{1}\right)$ where $\mathbb{X}$ is placed at "the point at infinity" of $D_{1}$ - the $k$-grading shift $\left(R_{2}, x_{2}, y_{2}\right)$ where $\mathbb{O}$ is placed at "the point at infinity" of $D_{2}$ This can be seen by dividing the arc from $\mathbb{X}$ to $\mathbb{O}$ into two pieces.

It follows that $h_{i}$ increases $\operatorname{gr}_{k}$ by an even integer in $[0, i+1]$.

Corollary 3.2. The $\mathrm{gr}_{h}, \mathrm{gr}_{q}$, and $\mathrm{gr}_{q}-\operatorname{gr}_{k}$ gradings on $\mathcal{C}$ endows $\left(\mathcal{C}_{\text {ftot }}, \delta_{\text {ftot }}\right)$ with the structure of a $(\mathbb{Z} \oplus \mathbb{Z} \oplus \mathbb{Z})$-filtered complex.

Proof. For each $(a, b, c) \in \mathbb{Z} \oplus \mathbb{Z} \oplus \mathbb{Z}$, define

$$
\mathcal{F}_{a, b, c}=\operatorname{Span}\left\{x \in \mathcal{C} \mid\left(\operatorname{gr}_{h}, \operatorname{gr}_{q}, \operatorname{gr}_{q-k}\right)(x) \geq(a, b, c)\right\}
$$

Lemma 3.1 tells us that $\delta_{f t o t}$ is non-decreasing with respect to the $\mathrm{gr}_{h}, \mathrm{gr}_{q}$, and $\mathrm{gr}_{q}-\mathrm{gr}_{k}$ gradings. So $\mathcal{F}_{a, b, c}$ is a subcomplex for each $(a, b, c) \in(\mathbb{Z} \oplus \mathbb{Z} \oplus \mathbb{Z})$. Moreover, $\left(a^{\prime}, b^{\prime}, c^{\prime}\right) \geq$ $(a, b, c) \in(\mathbb{Z} \oplus \mathbb{Z} \oplus \mathbb{Z})$ implies $\mathcal{F}_{a^{\prime}, b^{\prime}, c^{\prime}} \subset \mathcal{F}_{a, b, c}$, as desired.

Having considered the $\left(\mathrm{gr}_{h}, \mathrm{gr}_{q}, \mathrm{gr}_{k}\right)$ triple degree of all the elementary cobordisms (see Lemma 3.1, we can now define a two-parameter family of gradings $\operatorname{gr}_{r, t}$.

Definition 3.3. Let $x$ be a $\left(\mathrm{gr}_{h}, \mathrm{gr}_{k}, \mathrm{gr}_{j}\right)$-homogeneous basis element of $\mathcal{C}$. Let $t \in[0,1]$ and $r \in[0,1]$. Define

$$
\operatorname{gr}_{r, t}(x)=r \cdot \operatorname{gr}_{h}(x)+(1-r)\left(\operatorname{gr}_{q}(x)-t \cdot \operatorname{gr}_{k}(x)\right) .
$$

We note that $r=0$ corresponds to $\mathrm{gr}_{0, t}=\mathrm{gr}_{q}-t \cdot \mathrm{gr}_{k}$, which agrees with the $j_{t}$ grading in GLW17] (although their construction uses the Khovanov-Lee complex instead of the SarkarSeed-Szabó complex). The case $t=1$ corresponds to $\mathrm{gr}_{r, 1}=r \cdot \mathrm{gr}_{h}+(1-r) \cdot \mathrm{gr}_{q}$, which is precisely the filtration grading used in Sarkar-Seed-Szabó to define a family of concordance invariants that generalize the Rasmussen $s$ invariant.

Corollary 3.4. For every $r, t \in[0,1]$, the $\operatorname{gr}_{r, t}$ grading endows $\left(\mathcal{C}, \delta_{f t o t}\right)$ with the structure of a (discrete, bounded) $\mathbb{R}$-filtered complex equipped with a finite filtered graded basis.

Proof. Lemma 3.1 implies that $\delta_{f t o t}$ is non-decreasing with respect to the $\mathrm{gr}_{r, t}$ grading for each $r, t \in[0,1]$. It follows that for each $a \in \mathbb{R}$, the subcomplexes

$$
\mathcal{F}_{a}=\operatorname{Span}\left\{x \in \mathcal{C} \mid \operatorname{gr}_{r, t}(x) \geq a\right\}
$$

endow $\mathcal{C}$ with the structure of an $\mathbb{R}$-filtered complex. The finiteness of the distinguished filtered graded basis implies that the $\mathbb{R}$-filtration is discrete and bounded. 
Therefore, $\mathcal{C}$ admits a filtration grading given by

$$
g r_{r, t}(x)=\max \left\{i \in I \mid x \in \mathcal{F}_{i}\right\} .
$$

The homology $H_{*}(\mathcal{C})$ inherits a grading from $\mathcal{C}$ as follows.

Definition 3.5. Given $0 \neq[x] \in H_{*}(\mathcal{C})$, we define the $g r_{r, t}$ grading of $[x]$ to be

$$
g r_{r, t}([x])=\max _{y \in \mathcal{C}}\left\{g r_{r, t}(y) \mid[y]=[x]\right\} .
$$

Let $L \subset(A \times I)$ be an $l$-component annular link. By Proposition 5.4 of [SSS17], $H_{*}\left(\mathcal{C}_{f t o t}(L)\right)$ has rank $2^{l}$ and there are canonical generators $g(o)$ in canonical correspondence with orientations $o$ on $L$, just as in the the Bar-Natan [Tur06] and Lee [Lee05] theories. Given a link diagram $\mathcal{D}$ with an orientation, the canonical generator $g(o) \in \mathcal{C}_{f t o t}$ is given by the tensor product of labels $x_{i}$ or $1+x_{i}$ on the circles in the oriented resolution, assigned by the following rule. Fix the white-and-black checkerboard colroing of the oriented resolution where the unbounded region is white, and label circle $i$ with $x_{i}$ if it bounds a black region, or $1+x_{i}$ if it bounds a white region.

Definition 3.6. Let $r \in[0,1]$ and $t \in[0,1]$. Define $s_{r, t}(L, o)=\operatorname{gr}_{r, t}([g(o)])$.

We will show in part 1 of Theorem 3.15 that the $s_{r, t}(L, o)$ invariants are indeed oriented annular link invariants.

Example 3.7. We compute $s_{r, t}\left(\widehat{\mathbb{1}}_{n}, O\right)$ for the closure of the identity $n$-braid $\widehat{\mathbb{1}}_{n}$ with a choice of orientation $o$. Using the crossingless diagram for $\widehat{\mathbb{1}}_{n}$, we observe that $s_{r, t}\left(\widehat{\mathbb{1}}_{n}, o\right)=$ $\operatorname{gr}_{r, t}\left(v_{-} \otimes v_{-} \otimes \cdots \otimes v_{-}\right)=(1-r)(-n+t n)=-n(1-r)(1-t)$. In particular, $s_{r, t}\left(\widehat{\mathbb{1}}_{n}, o\right)$ is independent of the choice of orientation $o$ of $\widehat{\mathbb{1}}_{n}$.

Example 3.8. Let $\widehat{\mathbb{1}}_{1}^{+} \in \mathfrak{B}_{2}$ denote the closure of the positive Markov stabilization of the identity 1-braid. Given any choice of orientation $o$ on $\widehat{\mathbb{1}}_{1}^{+}$,

$$
s_{r, t}\left(\widehat{\mathbb{1}}_{1}^{+}, o\right)=(1-r)(-1+2 t)
$$

Let $\widehat{\mathbb{1}}_{1}^{-} \in \mathfrak{B}_{2}$ denote the closure of the negative Markov stabilization of the identity 1-braid. Given any choice of orientation $o$ on $\widehat{\mathbb{1}}_{1}^{-}$,

$$
s_{r, t}\left(\widehat{\mathbb{1}}_{1}^{-}, o\right)=(1-r)(-1)
$$

Remark 3.9 (Comparison with the Sarkar-Seed-Szabó concordance invariants). We regard the real-valued $s_{r, 0}(K, o)$ invariant (where $t=0$ ) as a continuous sibling of the Sarkar-SeedSzabó $2 \mathbb{Z}$-valued $s_{o}^{\mathcal{U}_{(r)}}(K)$ concordance invariant as follows. For $r \in[0,1]$, Sarkar-Seed-Szabó define the upright set

$\mathcal{U}_{(r)}=\{(h, q) \in \mathbb{Z} \times(2 \mathbb{Z} \times 1) \mid r \cdot h+(1-r) \cdot q>0$, or $[r \cdot h+(1-r) \cdot q=0$ and $q \geq 0]\}$.

Sarkar-Seed-Szabó introduce notation for shifts $\mathcal{U}[n]$ of an upright set $\mathcal{U}$ in the $\left(\operatorname{gr}_{h}, \operatorname{gr}_{q}\right)$ plane:

$$
(h, q) \in \mathcal{U}[n] \Longleftrightarrow(h, q-n) \in \mathcal{U}
$$

for any upright set $\mathcal{U}$. Sarkar-Seed-Szabó only allow shifts by even integers $n \in \mathbb{Z}$, but for purposes of comparison with our invariants, we extend the definition to allow for shifts by any real number $n \in \mathbb{R}$. Sarkar-Seed-Szabó define

$$
s_{o}^{\mathcal{U}}(K, o)=\max \left\{n \in 2 \mathbb{Z} \mid \mathcal{F}_{\mathcal{U}[n]} \mathcal{C}_{\text {ftot }}(K) \text { contains a rep. of }[g(o)]\right\}+2
$$


where $[g(o)]$ denotes the homology class of the generator $g(o)$.

In our case, the filtration level $\mathcal{F}_{n}^{r, 0}$ of $\mathcal{C}_{f t o t}$ defined by the grading $\operatorname{gr}_{r, 0}$ is the half-plane

$$
\mathcal{F}_{n}^{r, 0}=\{(h, q) \in \mathbb{R} \times \mathbb{R} \mid r \cdot h+(1-r) \cdot q \geq n\},
$$

where we abuse notation by conflating a filtration level of $\mathcal{C}$ with the half plane in $\left(\mathrm{gr}_{h}, \mathrm{gr}_{q}\right)$ space it is defined to be supported on. We see that

$$
\mathcal{F}_{n}^{r, 0} \cap(\mathbb{Z} \times(2 \mathbb{Z}+1))=\mathcal{U}_{(r)}\left[\frac{n}{1-r}\right] \cup\{(h, q) \mid r \cdot h+(1-r) \cdot q=n \text { and }(1-r) q<n\} .
$$

That is, our discretized filtration levels $\mathcal{F}_{n}^{r, 0} \cap(\mathbb{Z} \times(2 \mathbb{Z}+1))$ agree with the Sarkar-Seed-Szabó filtration levels $\mathcal{U}_{(r)}\left[\frac{n}{1-r}\right]$, with a different boundary condition.

Remark 3.10. If the annular link $L$ is unlinked from the unknotted axis $U$, then $s_{r, t}(L)=$ $s_{r, 0}(L)$ for all $t \in[0,1]$. Furthermore, if $[L]$ denotes the isotopy class of $L$ in $S^{3}$, then $s_{r, 0}(L, o)$ "agrees" with $s^{\left.\mathcal{U}_{(} r\right)}([L], o)$, by the relationship described in Remark 3.9.

Proof. Observe that all distinguished generators have $\mathrm{gr}_{k}=0$, in which case $g r_{r, t}=r \cdot \mathrm{gr}_{h}+$ $(1-r) \operatorname{gr}_{q}$.

3.2. Behavior under cobordisms. Let us now review the definitions of the maps on $\mathcal{C}_{\text {ftot }}$ associated to the elementary movie moves, and describe their $\left(\mathrm{gr}_{h}, \mathrm{gr}_{q}, \mathrm{gr}_{k}\right)$ tridegree in an annular context. For the non-annular case, just consider the $\left(\mathrm{gr}_{h}, \mathrm{gr}_{q}\right)$ bidegree. Actual computations are performed in Section 3.1, specifically in Lemma 3.1.

In all of these cases, we consider a diagrammatic cobordism $F: \mathcal{D} \rightarrow \mathcal{D}^{\prime}$. The cobordism map associated to the elementary move is a map $f: \mathcal{C}_{f t o t}(\mathcal{D}) \rightarrow \mathcal{C}_{\text {ftot }}\left(\mathcal{D}^{\prime}\right)$.

First of all, for annular isotopies and annular Reidemeister moves, the associated map is the canonical isomorphism of the target and source complexes. Thus the interesting moves are the elementary cobordisms.

To a birth, Sarkar-Seed-Szabó associate the map $a \mapsto a \otimes x_{+}$. Dually, to a death, SarkarSeed-Szabó associate the map $a \otimes x_{-} \mapsto a, a \otimes x_{+} \mapsto 0$. In the annular case, let $x_{ \pm}=v_{ \pm}$or $w_{ \pm}$depending on whether the circle is nontrivial or trivial, respectively.

To an annular saddle, we get the associated map from looking at the differential in the complex corresponding the link diagram $\mathcal{D}^{\prime \prime}$ with a crossing at the site of the saddle cobordism, so that $\mathcal{C}_{f t o t}\left(\mathcal{D}^{\prime \prime}\right)=\operatorname{cone}\left(\mathcal{C}_{f t o t}(\mathcal{D}) \rightarrow \mathcal{C}_{f t o t}\left(\mathcal{D}^{\prime}\right)[1]\{1\}\right)$ (see Remark 3.11). So the cobordism map $f$ looks like the sum of the components of the differential (the $d_{i}$ and $h_{i}$ ), except that the homological and quantum degrees are one less than that of the true components of the differential.

Remark 3.11. The chain map associated to a saddle cobordism is defined as a map $\mathcal{C}_{\text {ftot }}(\mathcal{D}) \rightarrow \mathcal{C}_{\text {ftot }}\left(\mathcal{D}^{\prime}\right)$. Following [BN02], we use the convention that $\mathcal{C}[a]\{b\}^{h, q}=\mathcal{C}^{h-a, q-b}$. We can visualize degree shift as follows: $\mathcal{C}[a]\{b\}$ is the result of grabbing $\mathcal{C}$ and moving it $a$ units along the $\mathrm{gr}_{h}$-axis and $b$ units along the $\mathrm{gr}_{q}$-axis.

We may now describe the filtration degrees of the elementary cobordism maps.

Proposition 3.12 (cf. GLW17] Prop. 2). For $r, t \in[0,1]$, the $\operatorname{gr}_{r, t}$ filtration degrees of Sarkar-Seed-Szabó chain map associated to

(1) an annular elementary saddle cobordism is $(1-r)(-1)$,

(2) an annular birth / death is $1-r$,

(3) a non-annular birth / death is $(1-r)(1-t)$, and 
(4) an annular Reidemeister move is 0.

Proof. First note that the homological filtration degree shift for all of these elementary cobordism maps is 0 . In the following, "bifiltration" means $\left(\mathrm{gr}_{q}, \mathrm{gr}_{k}\right)$ bifiltration. Below, we compute the lower bound on the degree shift under elementary cobordism maps on $\operatorname{gr}_{q}-\operatorname{tgr}_{k}$, so that the lower bound on the degree shift for $\operatorname{gr}_{r, t}$ is $(1-r)$ times that of $\operatorname{gr}_{q}-\operatorname{tgr}{ }_{k}$.

(1) Recall that $d_{f t o t}=\sum_{i \geq 1} d_{i}+h_{i}$, where

(a) $d_{i}=d_{i,-2}+d_{i, 0}+d_{i, 2}$, with $d_{1,2}=0$

(b) $h_{i}=h_{i, 0}+h_{i, 2}+h_{i, 4}+\cdots+h_{i, i+1}$

\begin{tabular}{||c|c|ccc||c||}
\hline & & $\operatorname{gr}_{h}$ & $\operatorname{gr}_{q}$ & $\operatorname{gr}_{k}$ & $\operatorname{gr}_{q}-t \mathrm{gr}_{k}$ \\
\hline \hline$i \geq 1$ & $d_{i,-2}$ & $i$ & $2 i-2$ & -2 & $2 i-2+2 t$ \\
$i \geq 1$ & $d_{i, 0}$ & $i$ & $2 i-2$ & 0 & $2 i-2$ \\
$i>1$ & $d_{i, 2}$ & $i$ & $2 i-2$ & 2 & $2 i-2-2 t$ \\
$i \geq 1, j \in[0, i+1]$ & $h_{i, j}$ & $i$ & $2 i$ & $j$ & $2 i-j t$ \\
\hline
\end{tabular}

Therefore, the lower bound for the $\operatorname{gr}_{q}-\operatorname{tgr}{ }_{k}$ shift is 0 .

Note that the filtrations degrees in the chart above take into account the quantum grading shift of +1 when we move along an edge, within a chain complex. The map associated to a saddle cobordism corresponds to such an edge map, but without this shift in quantum grading, so the lower bound for the $\operatorname{gr}_{q}-\operatorname{tgr}_{k}$ shift is -1 for a saddle cobordism.

(2) Let $\mathbb{W}$ denote the 2-dimensional vector space over $\mathbb{F}_{2}$ generated by two distinguished generators, at $\left(\mathrm{gr}_{q}, \mathrm{gr}_{k}\right)$ degrees $(1,0)$ and $(-1,0)$. This is the vector space underlying the chain complex for a single annular circle.

The birth of an annular circle corresponds to the inclusion map $\mathcal{C} \rightarrow \mathcal{C} \otimes \mathbb{W}$ which takes $x \mapsto x \otimes w_{+}$, and hence has bifiltration degree $(1,0)$.

The death of an annular circle corresponds to the projection map $\mathcal{C} \otimes \mathbb{W}$ which takes $x \otimes w_{+} \mapsto 0$ and $x \otimes w_{-} \mapsto x$, and hence also have bifiltration degree $(1,0)$ as well. (Note that one can think of $\operatorname{gr}_{q}\left(0 \in \mathbb{F}_{2}[x] /\left(x^{2}\right)\right)=\infty$.)

Therefore, the lower bound for the $\mathrm{gr}_{q}-\operatorname{tgr}_{k}$ shift is 1 .

(3) Similarly, let $\mathbb{V}$ be the 2-dimensional vector space underlying the chain complex for a single non-annular circle. This has two distinguished generators, at bifiltration degrees $(1,1)$ and $(-1,-1)$.

The nonannular birth map $\mathcal{C} \rightarrow \mathcal{C} \otimes \mathbb{V}$ sends $x \mapsto x \otimes v_{+}$and hence has bifiltration degree $(1,1)$.

The nonannular death map $\mathcal{C} \otimes \mathbb{V} \rightarrow \mathcal{C}$ sends $x \otimes v_{+} \mapsto 0$ and $x \otimes v_{-} \mapsto x$ and hence has bifiltration degree $(1,1)$ as well.

Therefore, the lower bound for the $\operatorname{gr}_{q}-t \operatorname{gr}_{k}$ shift is $1-t$.

(4) As in GLW17] Proposition 2, the chain homotopies that give Reidemeister equivalences do not interact with the annular axis, so the $\mathrm{gr}_{k}$ filtration degree of the annular cobordism ("annular" in the sense of a concordance) is 0.

The $\mathrm{gr}_{q}$ filtration degree is 0 by Section 4 of [SSS17].

For reference, here are the tridegrees associated to elementary cobordism maps: 
Corollary 3.13. Consider the components of the saddle cobordism map corresponding to the $d_{i}$ and $h_{i}$ maps which define the differential. For $d_{i}$, the $\operatorname{gr}_{h}$-degree is $i-1$, the $\operatorname{gr}_{q}$-degree is $2 i-3$, and the $\operatorname{gr}_{k}$-degree is $-2,0$, or 2 . For $h_{i}$, the $\operatorname{gr}_{h}$-degree is $i-1$, the $\operatorname{gr}_{q}$ degree is $2 i-1$, and the $\operatorname{gr}_{k}$ degree is an integer in $[0, i+1]$. The $\left(\mathrm{gr}_{h}, \mathrm{gr}_{q}, \mathrm{gr}_{k}\right)$ triple degree of the cobordism map is $(0,1,0)$ for annular births and deaths, and $(0,1,1)$ for non-annular births and deaths.

We now state the main properties of the two-dimensional family of annular link invariants $s_{r, t}(L, o)$. These features can be compared with corresponding properties of Grigsby-LicataWehrli's annular Rasmussen invariants $d_{t}$, cf. [GLW17, Theorem 1].

Definition 3.14. The wrapping number $\omega(L)$ of an annular link $L$ is the minimal number of (transverse) intersections between the image of $L$ in a diagram $\mathcal{D}(L)$ and an arc connecting $\mathbb{X}$ and $\mathbb{O}$, over all possible diagrams of $L$.

Theorem 3.15. Let $(L, o)$ be an oriented annular link. Let $r \in[0,1], t \in[0,1]$.

(1) For each pair $r, t \in[0,1], s_{r, t}(L, o)$ is an oriented annular link invariant.

(2) Suppose $L$ is a knot. Then $s_{0,0}(L, o)=s_{\mathbb{F}_{2}}(L, o)-1$, where $s_{\mathbb{F}_{2}}$ is Rasmussen's concordance invariant over $\mathbb{F}_{2}$ coefficients [Tur06, MTV07].

(3) For fixed $r$ (respectively $t$ ), the function $s_{r, t}(L, o)$ is piecewise-linear with respect to the variable $t$ (respectively $r$ ).

(4) Let $\omega$ be the wrapping number of $L$. Fix $r \in[0,1)$. Then for all $t_{0} \in[0,1)$

$$
\left(-\frac{1}{1-r}\right) \lim _{t \rightarrow t_{0}+} \frac{s_{r, t}(L, o)-s_{r, t_{0}}(L, o)}{t-t_{0}} \in\{-\omega,-\omega+2, \ldots, \omega-2, \omega\} .
$$

(5) Let $F:(L, o) \rightarrow\left(L^{\prime}, o^{\prime}\right)$ be an oriented cobordism between two nonempty, oriented links such that each component of $F$ has a boundary component in $L$. Let $a_{0}$ be the number of annular births or deaths, $a_{1}$ the number of saddles, and $b_{0}$ the number of non-annular births or deaths. Then

$$
s_{r, t}(L, o)-s_{r, t}\left(L^{\prime}, o^{\prime}\right) \leq(r-1)\left(a_{0}-a_{1}+b_{0}(1-t)\right) .
$$

If furthermore each component in $F$ has a boundary component in $L^{\prime}$ as well, then

$$
\left|s_{r, t}(L, o)-s_{r, t}\left(L^{\prime}, o^{\prime}\right)\right| \leq(r-1)\left(a_{0}-a_{1}+b_{0}(1-t)\right) .
$$

(6) $s_{r, t}$ is an annular concordance invariant.

Proof. (1) Let $\mathcal{D}$ and $\mathcal{D}^{\prime}$ be two annular link diagrams related by an annular Reidemeister move: that is, an isotopy or Reidemeister move that never crosses either of the marked points $\mathbb{X}$ or $\mathbb{O}$. Forgetting the location of the basepoints, Sarkar-Seed-Szabó show in Section 4 of [SSS17] that $\mathcal{C}_{\text {tot }}(\mathcal{D}) \simeq \mathcal{C}_{\text {tot }}\left(\mathcal{D}^{\prime}\right)$, i.e. are equal in the homotopy category of (bigraded) chain complexes $\mathcal{K}\left(\mathbb{F}_{2}[H, W]\right)$ over $\mathbb{F}_{2}[H, W]$. Thus they are also equal after applying the functor $\mathcal{K}\left(\mathbb{F}_{2}[H, W]\right) \rightarrow$ Filt which sets $H=1$ and $W=1$. Hence the $\operatorname{gr}_{h}$ and $\operatorname{gr}_{q}$ degrees of the chain homotopy equivalence between $\mathcal{C}_{f t o t}(\mathcal{D})$ and $\mathcal{C}_{\text {ftot }}\left(\mathcal{D}^{\prime}\right)$ are both 0 .

As Reidemeister moves are local, we can ensure that the small neighborhood where $\mathcal{D}$ differs from $\mathcal{D}^{\prime}$ is disjoint from the basepoints. 
It remains to check that the chain homotopy equivalence between the images $\mathcal{C}_{f t o t}(\mathcal{D})$ and $\mathcal{C}_{f t o t}\left(\mathcal{D}^{\prime}\right)$ in Filt (where $H, W=1$ throughout) has $\operatorname{gr}_{0,1}=\operatorname{gr}_{q}-\operatorname{gr}_{k}$ filtered degree 0 .

The chain homotopy equivalences are defined using cancellation (see [SSS17] Lemma 4.5). Cancellation of a cancellation data is a filtered chain homotopy equivalence if the component of the differential in the cancellation data is minimal in $\operatorname{gr}_{0,1}$ degree.

For Reidemeister I and II, it is easy to see that each cancellation step involves cancelling a differential between resolutions where all circles involved keep their trivial or nontrivial status, or are new trivial circles. Reidemeister III invariance is proven by reducing each complex to a smaller complex, and then noting that the smaller complexes are equivalent, by isotopy. So, it suffices to check that either of these reductions constitutes a filtered chain homotopy equivalence. Again, the cancellation data involve differentials that maintain the annular status of all circles.

Since the gr $_{q}$ degree of these cancellation data is known to be 0 , and by Lemma 3.1 . all components of the differential have $\mathrm{gr}_{0,1}$ degree at least 0 . Thus the components of the differential chosen for cancellation are indeed components with minimal $\mathrm{gr}_{0,1}$ degree.

(2) One can prove this using Lemma 5.5 in [SSS17], but perhaps the quickest proof relies on Sarkar-Seed-Szabó's invariants. When $r=0, \mathcal{U}_{(0)}[n]$ and $\mathcal{F}_{n}^{0,0}$ agree on the lattice $\mathbb{Z} \times(2 \mathbb{Z}+1)$. Note that the quantum grading of any generator in the Khovanov complex for a knot is an odd integer. Now Sarkar-Seed-Szabó's $s_{o}^{\mathcal{U}_{(0)}}(K)$, which they show is equal to $s_{\mathbb{F}_{2}}$, is the first even integer for which the filtration level $\mathcal{F}_{\mathcal{U}_{(0)}}[n]$ does not contain a representative of $[g(o)]$. Hence $s_{0,0}(K, o)=\operatorname{gr}_{q}[g(o)]$ must be the odd integer $s_{o}^{\mathcal{U}_{(0)}}(K)-1=s_{\mathbb{F}_{2}}-1$.

(3) For fixed $r$ (resp. $t$ ), the function $s_{r, t}(K, o)$ is piecewise because the complex $\mathcal{C}_{\text {ftot }}$ is finitely generated. Along intervals on which the same representative cycle $x \in[g(o)]$ achieves the maximum grading $g r_{r, t}(x)=g r_{r, t}[g(o)]$, the values $\operatorname{gr}_{h}(x), \operatorname{gr}_{q}(x), \operatorname{gr}_{k}(x)$, and $r$ (resp. $t$ ) are constant.

(4) Suppose $[y]$ is a nontrivial homology class. Then $g r_{r, t}[y]$ is achieved by some representative cycle $y \in[y]$, i.e. $g r_{r, t}(y)=g r_{r, t}[y]$. Write $y=\sum_{i} y_{i}$ where each $y_{i}$ is a distinguished generator. Then $g r_{r, t}(y)=\min _{i}\left\{g r_{r, t}\left(y_{i}\right)\right\}$. So, it suffices to prove the statement for distinguished generators.

Let $x$ be a distinguished generator. Then

$$
g r_{r, t}(x)=r \cdot \operatorname{gr}_{h}(x)+(1-r) \cdot\left(\operatorname{gr}_{q}(x)-t \cdot \operatorname{gr}_{k}(x)\right)
$$

SO

$$
\lim _{t \rightarrow t_{0}^{+}} \frac{g r_{r, t}(x)-\operatorname{gr}_{r, t_{0}}(x)}{t-t_{0}}=(1-r) \cdot\left(-\operatorname{gr}_{k}(x)\right) .
$$

Now the state of $x$ has at most $\omega v$-circles, each of which contributes \pm 1 (for $v_{ \pm}$, respectively) to $\operatorname{gr}_{k}(x)$, so $\operatorname{gr}_{k}(x) \in\{-\omega,-\omega+2, \ldots, \omega-2, \omega\}$.

(5) Let $\phi_{F}$ denote the chain map associated to the cobordism $F$, and let $\phi_{F}^{*}$ be the induced map on homology. Let $x \in[g(0)]$ such that $g r_{r, t}(x)=g r_{r, t}[g(0)]=s_{r, t}(L, o)$. By Proposition 3.12,

$$
g r_{r, t}\left(\phi_{F}(x)\right) \geq s_{r, t}(L, o)+(1-r)\left(a_{0}-a_{1}+b_{0}(1-t)\right) .
$$



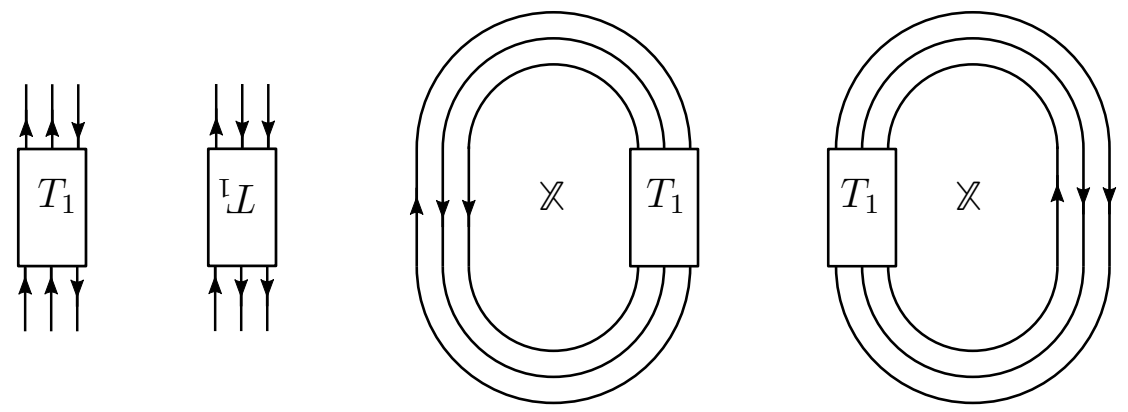

Figure 6. From left to right: the tangles $T_{1}$ and $\theta T_{1}$ in $\mathcal{T}_{3,3}^{\wedge}$ and their closures $\widehat{T}_{1}$ and $\widehat{\theta T_{1}}$, respectively.

By asserting that every component of $F$ has boundary on $(L, o)$, we assure that $\phi_{F}^{*}[g(o)]$ is (a nonzero multiple of) $\left[g\left(o^{\prime}\right)\right]$. (See BN-2 of Proposition 5.3 in [SSS17] and references therein.) Hence $\phi_{F}(x) \in\left[g\left(o^{\prime}\right)\right]$, so $g r_{r, t}\left(\phi_{F}(x)\right) \leq g r_{r, t}\left[g\left(o^{\prime}\right)\right]=s_{r, t}\left(L^{\prime}, o^{\prime}\right)$. Putting these two inequalities together, we obtain

$$
s_{r, t}\left(L^{\prime}, o^{\prime}\right) \geq s_{r, t}(L, o)+(1-r)\left(a_{0}-a_{1}+b_{0}(1-t)\right)
$$

and rearrange to obtain the desired inequality. To obtain the statement with absolute value, consider the opposite cobordism $-F:\left(L^{\prime}, o^{\prime}\right) \rightarrow(L, o)$ as well.

(6) Now consider the case where there is an annular concordance $F$ between $(L, o)$ and $\left(L^{\prime}, o^{\prime}\right)$. In particular, $(L, o)$ and $\left(L^{\prime}, o^{\prime}\right)$ each have $l$ components, $F$ a disjoint union of $l$ annuli, each with one boundary component in $L$ and the other in $L^{\prime}$. Recalling the notation from Section 2.2 , since $F$ is an annular concordance, $F$ is disjoint from $U \times I \subset S^{3} \times I$. Hence $b_{0}=0$, and $a_{0}=a_{1}$, so we have $\left|s_{r, t}(L, o)-s_{r, t}\left(L^{\prime}, o^{\prime}\right)\right| \leq 0$.

Remark 3.16. The one-parameter family of invariants $s_{r, 0}$ for $r \in[0,1]$ is related to the Sarkar-Seed-Szabó generalized Rasmussen invariants (see Remark 3.9). The definition of the one-parameter family of $s_{0, t}$ invariants resembles Grigsby-Licata-Wehrli's annular $d_{t}$ invariants, which specialize to Rasmussen's $s$-invariant with $\mathbb{Q}$ coefficients (at $t=0$ and 2 ).

Remark 3.17. Cotton Seed [LS14, Remark 6.1] has found examples where $s_{\mathbb{F}_{2}}(K) \neq s_{\mathbb{Q}}(K)$.

3.3. Tangle closures. Viewing annular links as tangle closures, we can relate the annular horizontal composition with the non-annular isotopy type of their (vertical) tangle composition by a sequence of 1-handle additions.

Before studying the behavior of $s_{r, t}$ under such an operation, we need a few topological definitions. See Figure 6 for examples.

Let $\mathcal{T}_{n, n}^{\wedge}$ be the set of oriented $(n, n)$-tangles whose top and bottom strands agree (i.e. the tangle can be closed up). Composition of $T_{1} \cdot T_{2}$ is given by stacking, if orientations on the ends permit this. Let $\widehat{T}$ denote the closure of $T$. If we view $\widehat{T} \subset A \times I$ as the annular closure of $T$, there is an ambiguity: we set the convention that the basepoint $\mathbb{X}$ is located directly to the left of $T$, and that $\widehat{T}$ is then closed around $\mathbb{X}$, so that in the diagram of $\widehat{T}, T$ is situated on the right.

Define an involution $\theta$ on annular links which swaps $\widehat{T}$ with the result of the other convention: diagrammatically, $\theta$ is equivalent to swapping the basepoints $\mathbb{X}$ and $\mathbb{O}$. For $T \in \mathcal{T}_{n, n}^{\wedge}$, define $\theta$ as the involution that rotates $T$ by $180^{\circ}$. Observe that $\theta \widehat{T}=\widehat{\theta T}$. 

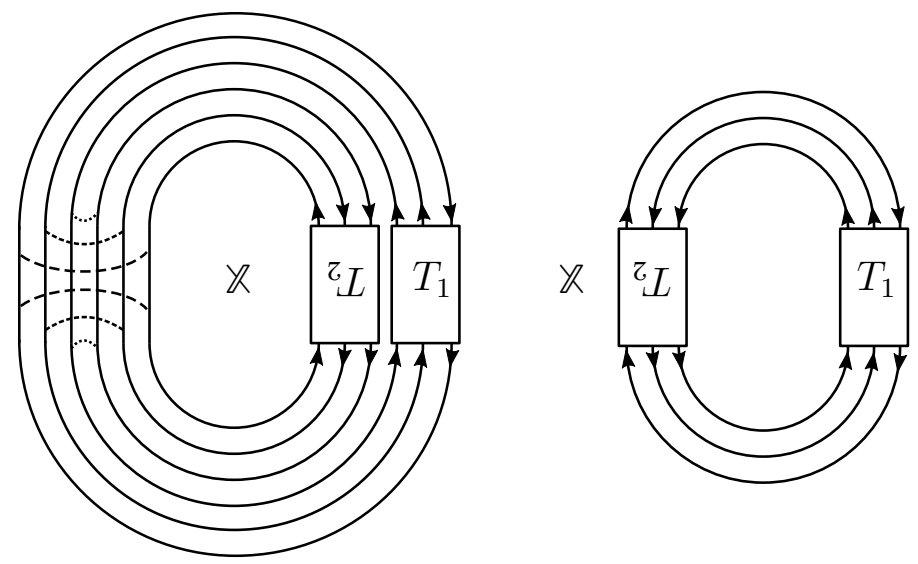

FiguRE 7 . The dotted lines on the left indicate the three saddle cobordisms which transform $\widehat{\theta T_{2}} \sqcup \widehat{T}_{1}$ into $\widehat{T_{1} T_{2}}$, shown on the right.

For an annular link $L$, let $[L]$ denote its (nonannular) isotopy class in $S^{3}$. Recall (from Remark 3.10) that if an annular link $L$ is disjoint from the axis (diagrammatically, $L$ is situated in a disk disjoint from an arc connecting $\mathbb{O}$ and $\mathbb{X})$, then $s_{r, t}(L)=s_{r, 0}(L)$ for all $t$. With this in mind, we write $s_{r}([L])=s_{r, 0}(L)$.

Lemma 3.18. Let $T_{1}, T_{2} \in \mathcal{T}_{n, n}^{\wedge}$. Then

$$
\left|s_{r}\left(\left[\widehat{T_{1} \cdot T_{2}}\right]\right)-s_{r, t}\left(\widehat{T}_{1} \sqcup \widehat{\theta T_{2}}\right)\right| \leq(1-r) n .
$$

Proof. By adding $n$ 1-handles to $\widehat{T}_{1} \sqcup \widehat{\theta T_{2}}$, we obtain the nonannular representative of $\widehat{T_{1} \cdot T_{2}}$ (see Figure 7). Then inequality then follows from Theorem 3.15 part 5 .

Lemma 3.19. Let $T \in \mathcal{T}_{n, n}^{\wedge}$. Then for all $r, t \in[0,1], s_{r, t}(\widehat{T})=s_{r, t}(\widehat{\theta T})$.

Proof. In the hypotheses of Lemma 3.18, let $T_{1}$ be the identity tangle $\mathbb{1}_{n}$ and let $T_{2}=T$. Combining with Proposition 3.30 , we obtain the inequality

$$
\left|s_{r}\left(\left[\widehat{T}_{2}\right]\right)-s_{r, t}\left(\widehat{T}_{1}\right)-s_{r, t}\left(\widehat{\theta T_{2}}\right)\right| \leq(1-r) n .
$$

Now obtain a similar inequality by replacing $T_{2}$ with $\theta T_{2}$ and observing that $\widehat{\theta \mathbb{1}_{n}}=\widehat{\mathbb{1}_{n}}$ and that $\left[\widehat{T}_{2}\right]=\left[\widehat{\theta T_{2}}\right]$ :

$$
\left|s_{r}\left(\left[\widehat{T}_{2}\right]\right)-s_{r, t}\left(\widehat{T}_{1}\right)-s_{r, t}\left(\widehat{T}_{2}\right)\right| \leq(1-r) n
$$

Resolving the absolute values in two different ways and combining these two inequalities forces $s_{r, t}\left(\widehat{\theta T_{2}}\right)-s_{r, t}\left(\widehat{T}_{2}\right)=0$.

Theorem 3.20. Let $T_{1}, T_{2} \in \mathcal{T}_{n, n}^{\wedge}$ be two composable tangles. Then for $r, t \in[0,1]$,

$$
\left|s_{r}\left(\left[\widehat{T_{1} \cdot T_{2}}\right]\right)-s_{r, t}\left(\widehat{T}_{1} \sqcup \widehat{T}_{2}\right)\right| \leq(1-r) n .
$$

In particular,

$$
\left|s_{r}\left(\left[\widehat{T_{1} \cdot T_{2}}\right]\right)-s_{r}\left(\left[\widehat{T_{1} \cdot \theta T_{2}}\right]\right)\right| \leq 2(1-r) n .
$$

(Note that $\left[\widehat{T_{1} \cdot \theta T_{2}}\right]=\left[\widehat{\theta T_{1} \cdot T_{2}}\right]$, as $\theta$ acts as identity on links in $S^{3}$.)

Proof. The first inequality follows by applying Lemma 3.19 to the statement of Lemma 3.18 . The second inequality follows from the first, since both $s_{r}\left(\widehat{T_{1} \cdot T_{2}}\right)$ and $s_{r}\left(\widehat{T_{1} \cdot \theta T_{2}}\right)$ are related to $s_{r, t}\left(\widehat{T}_{1} \sqcup \widehat{T}_{2}\right)=s_{r, t}\left(\widehat{T}_{1}\right)+s_{r, t}\left(\widehat{T}_{2}\right)=s_{r, t}\left(\widehat{T}_{1}\right)+s_{r, t}\left(\widehat{\theta T_{2}}\right)$. 
3.4. Applications to braids and more properties. The annular Sarkar-Seed-Szabó invariants are well-suited to studying annular braid closures equipped with their braid-like orientation. In fact, we will see many similarities in these applications to the annular $d_{t}$ invariants of Grigsby-Licata-Wehrli [GLW17].

Let $\sigma \in \mathfrak{B}_{n}$ be an $n$-strand braid and $\widehat{\sigma} \subset A \times I$ its annular closure. The braid-like orientation $\sigma_{\uparrow}$ of $\widehat{\sigma}$ is the one whose strands all wind positively around the braid axis. Its diagram winds counterclockwise about $\mathbb{X}$ in $S^{2} \backslash\{\mathbb{O}, \mathbb{K}\}$. We shall abbreviate $s_{r, t}\left(\widehat{\sigma}, o_{\uparrow}\right)$ to $s_{r, t}(\widehat{\sigma})$.

Lemma 3.21. Let $\sigma \in \mathfrak{B}_{n}$ have writhe $w$. Then

$$
(1-r)(w-(1-t) n) \leq s_{r, t}(\widehat{\sigma})
$$

for all $r \in[0,1], t \in[0,1]$.

Proof. We calculate from the definitions:

$$
\operatorname{gr}_{h}\left(g\left(o_{\uparrow}\right)\right)=0, \quad \operatorname{gr}_{q}\left(g\left(o_{\uparrow}\right)\right)=-n+w, \quad \text { and } \operatorname{gr}_{k}\left(g\left(o_{\uparrow}\right)\right)=-n .
$$

Thus, for all $r \in[0,1], t \in[0,1]$,

$$
g r_{r, t}\left(g\left(o_{\uparrow}\right)\right)=(1-r)(w-(1-t) n) \leq g r_{r, t}\left(\left[g\left(o_{\uparrow}\right)\right]\right)=s_{r, t}(\widehat{\sigma})
$$

as desired.

When $r=0$, the lower bound on $s_{r, t}$ given by Lemma 3.21 is analogous to lower bound on the $d_{t}$ invariant of [GLW17, Lemma 6]. In particular, we recover a " $s_{\mathbb{F}_{2}}$-Bennequin inequality" (proven by Plamenevskaya [Pla06, Proposition 4] and Shumakovitch [Shu07, Lemma 4.C] originally for $s$ over $\mathbb{Q}$ coefficients).

Corollary 3.22. Let $\sigma \in \mathfrak{B}_{n}$ have writhe $w$. Then

$$
\operatorname{sl}(\widehat{\sigma}) \leq s_{\mathbb{F}_{2}}(\widehat{\sigma})-1
$$

where $s_{\mathbb{F}_{2}}$ is the Rasmussen concordance invariant over $\mathbb{F}_{2}$ and $\operatorname{sl}(\widehat{\sigma})=-n+w$ is the selflinking number of the transverse link represented by $\widehat{\sigma}$.

Proof. We specialize the result of Lemma 3.21 to $r=0$ and $t=0$.

By work of Cotton Seed (see Remark 3.17), in some cases this produces a stronger upper bound on the self-linking number than the "s-Bennequin inequality" for $s$ over $\mathbb{Q}$. Moreover, Lemma 3.21 gives rise to a new family of Bennequin-type inequalities.

Corollary 3.23. Let $\sigma \in \mathfrak{B}_{n}$ have writhe $w$. Then

$$
(1-r) \operatorname{sl}(\widehat{\sigma}) \leq s_{r, 0}(\widehat{\sigma})
$$

for all $r \in[0,1]$.

Proof. We specialize the result of Lemma 3.21 to $t=0$.

Furthermore, Theorem 3.24 shows that for quasipositive braid closures the $s_{\mathbb{F}_{2}}$-Bennequin bound is sharp.

Theorem 3.24. If $\sigma$ is a quasipositive braid of index $n$ and writhe $w \geq 0$, we have

$$
s_{r, t}(\widehat{\sigma})=(1-r)(w-(1-t) n)
$$

for all $r \in[0,1]$ and $t \in[0,1]$. 
Proof. Lemma 3.21 gives us the lower bound

$$
(1-r)(w-(1-t) n) \leq s_{r, t}(\widehat{\sigma}) .
$$

To obtain the upper bound, note that if $\widehat{\sigma}$ is quasipositive, there is an oriented (annular) cobordism $F$ from $\widehat{\sigma}$ to $\widehat{\mathbb{1}}_{n}$ obtained by performing an orientable saddle cobordism near each quasipositive generator of $\sigma$ as in [GLW17, Theorem 2] and [Pla06, Fig. 7], and each component of this cobordism has a boundary on $\widehat{\sigma}$. Using the crossingless diagram for $\widehat{\mathbb{1}}_{n}$, we have

$$
s_{r, t}\left(\widehat{\mathbb{1}}_{n}\right)=-(1-r)(1-t) n .
$$

Part (5) of Theorem 3.15 tells us that $s_{r, t}(\widehat{\sigma})-s_{r, t}\left(\widehat{\mathbb{1}}_{n}\right) \leq(1-r) w$. Thus,

$$
s_{r, t}(\widehat{\sigma}) \leq-(1-r)(1-t) n+(1-r) w
$$

as desired.

Theorem 3.25. Suppose $\sigma \in \mathfrak{B}_{n}$ has writhe $w$. Then $s_{r, 1}(\widehat{\sigma})=(1-r) w$.

Proof. Let $\mathcal{D}$ be a diagram of the annular braid closure $\widehat{\sigma} \subset A \times I$ and let $\mathcal{C}$ denote the graded vector space underlying the Sarkar-Seed-Szabó complex. The vector space $\mathcal{C}$ is generated by resolutions of $\mathcal{D}$ whose circles are labeled by $a=v_{-}+v_{+}$or $b=v_{-}$. The set of $a / b$ markings of resolutions of $\mathcal{D}$ forms a basis for $\mathcal{C}$. (This is not a $\mathbb{Z} \oplus \mathbb{Z} \oplus \mathbb{Z}$ filtered basis for $\left(C, \partial_{\text {ftot }}\right)$ ). We will denote the set of these generators by $S$. We will partition $S$ into three subsets:

(1) $S_{1}=\{g(o)\}$

(2) $S_{2}=\left\{x \in\left(S \backslash S_{1}\right) \mid x\right.$ is a labeling of the braid-like resolution of $\left.\mathcal{D}\right\}$

(3) $S_{3}=S \backslash\left(S_{1} \cup S_{2}\right)$

(Recall that the braid-like resolution of $\mathcal{D}$ is the oriented resolution for the braid-like orientation $o_{\uparrow}$.) Corresponding to this partition of $S$, there is a direct sum decomposition of $\mathcal{C}$ into subspaces: $\mathcal{C}=V_{1} \oplus V_{2} \oplus V_{3}$, with $V_{i}=\operatorname{Span}\left(S_{i}\right)$.

Let

$$
\begin{aligned}
& p: \mathcal{C} \rightarrow V_{1} \oplus V_{2} \\
& q: V_{1} \oplus V_{2} \rightarrow V_{1}
\end{aligned}
$$

denote the projection maps. Note that $p$ and $q$ satisfy the following properties.

(1) With respect to the $\operatorname{gr}_{r, 1}$-grading on $\mathcal{C}, p$ is a grading-preserving map of graded vector spaces.

(2) $q \circ p$ is a chain map.

(3) $(q \circ p)(g(o))=g(o)$.

We can now prove the following claims.

Claim 1. If $z \in \mathcal{C}$ is a cycle satisfying $[z]=[g(o)]$, then $p(z) \neq 0 \in \mathcal{C}$.

Proof. Suppose $z=g(o)+\delta_{f t o t}(x)$ for some $x \in \mathcal{C}$. Hence

$$
(q \circ p)(z)=(q \circ p)(g(o))+(q \circ p)\left(\delta_{f t o t} x\right)=g(o)+\delta_{f t o t}(q \circ p)(x) .
$$

Thus $[(q \circ p)(z)]=[g(o)]$, and since $g(o)$ is nonzero, this shows that $p(z)$ is nonzero.

Claim 2. $s_{r, 1}(\widehat{\sigma}) \leq w$. 
Proof. Let $z$ be a representative of $[g(o)]$ for which $\operatorname{gr}_{r, 1}(z)=\operatorname{gr}_{r, 1}[g(o)]$. Then $p(z)$ is nonzero by Claim 1. Since all elements in $V_{1} \oplus V_{2}$ have $\operatorname{gr}_{r, 1}=(1-r) w$, we have $\operatorname{gr}_{r, 1}(p(z))=(1-r) w$. Since $p$ is graded as a map of $\operatorname{gr}_{r, 1}$-graded vector spaces, this implies

$$
s_{r, 1}(\widehat{\sigma})=\operatorname{gr}_{r, 1}(z) \leq \operatorname{gr}_{r, 1}(p(z))=(1-r) w .
$$

On the other hand,

$$
s_{r, 1}(\widehat{\sigma})=\operatorname{gr}_{r, 1}[g(o)] \geq \operatorname{gr}_{r, 1}(g(o))=(1-r) w,
$$

so $s_{r, 1}(\widehat{\sigma})=(1-r) w$ as desired.

Recall from [GLW17] that the obstruction to being quasipositive from $d_{t}$ is no more sensitive than the one coming from the sharpness of the $s$-Bennequin bound. Similarly, the obstruction to being quasipositive from $s_{0, t}$ is no more sensitive than the one coming from the sharpness of the $s_{\mathbb{F}_{2}}$-Bennequin bound.

Corollary 3.26. If $\sigma \in \mathfrak{B}_{n}$ has writhe $w$, then

$$
s_{0, t}(\widehat{\sigma})=w-(1-t) n \quad \Longleftrightarrow \quad \operatorname{sl}(\widehat{\sigma})=s_{\mathbb{F}_{2}}(\widehat{\sigma})-1 .
$$

Proof. Recalling that $\operatorname{sl}(\widehat{\sigma})=-n+w$, the forward implication follows from setting $r=0$ and $t=0$ and applying part 2 of Theorem 3.15 .

For the converse, note the following:

(1) The function $s_{0, t}$ is piecewise linear (by part 3 of Theorem 3.15).

(2) The slope of $s_{0, t}$ with respect to $t$ is bounded above by $n$ (by part 4 of Theorem 3.15).

(3) By the hypothesis, $s_{0,0}=-n+w$.

(4) We have $s_{0,1}=w$ by Theorem 3.25 .

These observations immediately imply the reverse implication.

Let

$$
m_{r_{0}, t_{0}}(L, o)=\lim _{t \rightarrow t_{0}^{+}} \frac{s_{r, t}(L, o)-s_{r, t_{0}}(L, o)}{t-t_{0}}
$$

We have a sufficient condition for a braid conjugacy class to be right-veering. This property is analogous to that of the $d_{t}$ invariant (cf. [GLW17] Theorem 4). The bounds on $m_{r, t}(L, o)$ by part 4 of Theorem 3.15 will be essential in the following theorem.

Theorem 3.27. Let $\sigma \in \mathfrak{B}_{n}$. Fix $r=r_{0}<1$. If $s_{r_{0}, t}(\widehat{\sigma})$ attains maximal slope at some $t=t_{0}<\frac{1}{2}$, that is, $m_{r_{0}, t_{0}}(\widehat{\sigma})=n$ for some $t_{0} \in\left[0, \frac{1}{2}\right)$, then $\sigma$ is right-veering.

Proof. The proof relies on Hubbard-Saltz's annular invariant $\kappa$ [HS16], which has the following property: if $\sigma \in B_{n}$ is not right-veering, then $\kappa(\widehat{\sigma})=2$.

First, we review the definition of $\kappa$ and explain how it is related to the Sarkar-Seed-Szabó complex. Throughout this proof, let $G$ denote the set of distinguished generators of the Khovanov complex $\mathcal{C}=\langle g \in G\rangle$, over $\mathbb{F}_{2}$ (which is equal to $\mathcal{C}_{f t o t}$ as a vector space). Let $\sigma \in B_{n}$. A generic cycle can be thought of as a subset of $G$, as we are working over $\mathbb{F}_{2}$.

Recall from Lemma 3.1 that $\left(\mathcal{C}, d_{1}\right)$ is $\operatorname{gr}_{k}$ filtered: let $\mathcal{F}_{c}(\mathcal{C})=\left\langle x \in G \mid \operatorname{gr}_{k}(x) \leq c\right\rangle$; the $\mathrm{gr}_{k}$ degree of a component of $d_{1}$ is either 0 or -2 . 
Let $\mathbf{v}_{-} \in G$ denote Plamenevskaya's cycle, the distinguished generator at the braidlike resolution with all circles labeled with $v_{-}$. Hubbard and Saltz define

$$
\kappa(\widehat{\sigma})=n+\min \left\{c \mid\left[\mathbf{v}_{-}\right]=0 \in H_{*}\left(\mathcal{F}_{c}(\mathcal{C})\right)\right\} .
$$

We record a few computations for future use:

- If $a=\operatorname{gr}_{q}(x)$ and $b=\operatorname{gr}_{q-k}(x)$, then $\operatorname{gr}_{k}(x)=a-b$ and $\operatorname{gr}_{0, t}(x)=(1-t) a+t b$.

- Note that $\operatorname{gr}_{h}\left(\mathbf{v}_{-}\right)=0$. We have $\operatorname{gr}_{q}\left(\mathbf{v}_{-}\right)=-n+w, \operatorname{gr}_{k}\left(\mathbf{v}_{-}\right)=-n$, so

$$
\operatorname{gr}_{r, t}\left(\mathbf{v}_{-}\right)=(1-r)(-n(1-t)+w) \text {. }
$$

Note that $\mathbf{v}_{-}$is the unique generator with the minimum possible $\operatorname{gr}_{k}$-grading, namely $\operatorname{gr}_{k}\left(\mathbf{v}_{-}\right)=-n$, so it indeed is a cycle in $\left(\mathcal{C}, d_{1}\right)$, and it is the unique cycle with $\left(\operatorname{gr}_{q}, \operatorname{gr}_{q-k}\right)$ bigrading $(-n+w, w)$.

Suppose, by way of contradiction, that $\kappa(\widehat{\sigma})=2$ and there exists a time $t_{0} \in[0,1 / 2)$ at which $m_{r_{0}, t_{0}}(\widehat{\sigma})=\left(1-r_{0}\right) n$.

Since $\kappa(\widehat{\sigma})=2$, there is some chain $\theta \in \mathcal{C}$ such that $d_{1}(\theta)=\mathbf{v}_{-} \in \mathcal{F}_{-n}$. Since $d_{1}$ shifts $\operatorname{gr}_{k}$ degree by 0 or $-2, \theta \in \mathcal{F}_{-n+2}$, but since $\mathbf{v}_{-}$is the unique cycle in $\mathcal{F}_{-n}$, we know that in fact $\operatorname{gr}_{k}(\theta)=-n+2$, and so has homogeneous $\left(\operatorname{gr}_{h}, \operatorname{gr}_{q}, \operatorname{gr}_{q-k}\right)$ trigrading $(-1,-n+w, w-2)$.

Since $m_{r_{0}, t_{0}}(\widehat{\sigma})=\left(1-r_{0}\right) n$, there is a cycle $\xi \subset G$ for which $\operatorname{gr}_{r_{0}, t_{0}}(\xi)=s_{r_{0}, t_{0}}(\widehat{\sigma})$ and $\operatorname{gr}_{k}(\xi)=-n$. Hence $\mathbf{v}_{-} \in \xi$, and is one of the generators in $\xi$ whose gradings determine $s_{r, t}$ at $\left(r_{0}, t_{0}\right)$, i.e.

$$
\min _{x \in \xi}\left\{\operatorname{gr}_{r_{0}, t_{0}}(x)\right\}=\operatorname{gr}_{r_{0}, t_{0}}\left(\mathbf{v}_{-}\right)=\left(1-r_{0}\right)\left(-n\left(1-t_{0}\right)+w\right) .
$$

At this point, there are a priori two possibilities. Let $\xi^{\prime}=\xi \backslash\left\{\mathbf{v}_{-}\right\}$.

(1) There is a generator $x^{\prime} \in \xi^{\prime}$ achieving this minimum, i.e.

$\operatorname{gr}_{r_{0}, t_{0}}\left(x^{\prime}\right)=r_{0}(-1)+\left(1-r_{0}\right)\left(-n+w-t_{0} \operatorname{gr}_{k}\left(x^{\prime}\right)\right)=\left(1-r_{0}\right)\left(-n\left(1-t_{0}\right)+w\right)$.

Then $s_{r_{0}, t_{0}}$ can be computed by measuring $x^{\prime}$ as well. But this forces $\operatorname{gr}_{k}\left(x^{\prime}\right)=-n$, contradicting $x^{\prime} \notin \mathcal{F}(-n)$.

(2) Otherwise, for all $x^{\prime} \in \xi^{\prime}$

$$
\operatorname{gr}_{r_{0}, t_{0}}\left(x^{\prime}\right)>\operatorname{gr}_{r_{0}, t_{0}}\left(\mathbf{v}_{-}\right) \text {. }
$$

Recall that $\delta_{f t o t}=\sum_{i=1}^{\infty} d_{i}+\sum_{i=1}^{\infty} h_{i}$. Let $\xi^{\prime \prime}=\xi+\left(\sum_{i=1}^{\infty} d_{i}+\sum_{i=1}^{\infty} h_{i}\right) \theta$, so that $\left[\xi^{\prime \prime}\right]=[g(o)] \in H_{*}\left(\mathcal{C}_{f t o t}\right)$. Since $\mathbf{v}_{-}=d_{1}(\theta)$,

$$
\xi^{\prime \prime}=\xi^{\prime}+\left(\sum_{i=2}^{\infty} d_{i}+\sum_{i=1}^{\infty} h_{i}\right) \theta .
$$

From Lemma 3.1, one computes that $\sum_{i=2}^{\infty} d_{i}+\sum_{i=1}^{\infty} h_{i}$ increases $\operatorname{gr}_{h}$ by at least $1, \mathrm{gr}_{q}$ by at least 2 , and $\operatorname{gr}_{q-k}$ by at least 0 . Since $\left(\operatorname{gr}_{h}, \operatorname{gr}_{q}, \operatorname{gr}_{q-k}\right)(\theta)=(-1,-n+w, w-2)$,

$$
\operatorname{gr}_{r_{0}, t_{0}}\left(\left(\sum_{i=2}^{\infty} d_{i}+\sum_{i=1}^{\infty} h_{i}\right) \theta\right) \geq\left(1-r_{0}\right)\left(-n\left(1-t_{0}\right)+w+2-4 t_{0}\right),
$$

which is strictly greater than $\mathrm{gr}_{r_{0}, t_{0}}\left(\mathbf{v}_{-}\right)=\left(1-r_{0}\right)\left(-n\left(1-t_{0}\right)+w\right)$ when $\left(1-r_{0}\right)(2-$ $\left.4 t_{0}\right)>0$, i.e. when $r_{0} \in[0,1)$ and $t_{0} \in[0,1 / 2)$, contradicting the assumption that $\operatorname{gr}(\xi)=\operatorname{gr}_{r_{0}, t_{0}}[g(o)]$. 
Remark 3.28. The upper bound $t<\frac{1}{2}$ in Theorem 3.27 is reminiscent of the upper bound $t<1$ on the slope of $\Upsilon$ in Theorem 1.0.4 of [He18].

Proposition 3.29. Let $\sigma \in \mathfrak{B}_{n}$ have writhe $w$. If $m_{r, t_{0}}(\widehat{\sigma})=(1-r) n$ for some $t_{0} \in[0,1)$, then $m_{r, t}=(1-r) n$ for all $t \in\left[t_{0}, 1\right)$.

Proof. Recall that there is a unique distinguished basis vector, Plamenevskaya's cycle $\mathbf{v}_{-}$, with $k$-grading $-n$. In view of part 4 of Theorem 3.15, this implies that

$$
\operatorname{gr}_{r, t_{0}}([g(o)])=\operatorname{gr}_{r, t_{0}}\left(\mathbf{v}_{-}\right)=(1-r)\left((-n+w)+n t_{0}\right) .
$$

Recall that $s_{r, 1}(\widehat{\sigma})=(1-r) w$ by Theorem 3.25 and

$$
m_{r, t} \leq(1-r) n
$$

by part 4 of Theorem 3.15. Therefore, the slope $m_{r, t}=(1-r) n$ for all $t \in[0,1)$.

We will show that the annular $s_{r, t}$ invariants are additive under horizontal composition and behave well under orientation reversal. These properties are also shared with the $d_{t}$ invariants of GLW17.

Proposition 3.30. Let $(L, o),\left(L^{\prime}, o^{\prime}\right) \subset A \times I$ and let $(L, o) \sqcup\left(L^{\prime}, o^{\prime}\right) \subset A \times I$ denote their annular composition, as in GLW17, Figure 2]. Then for all $r \in[0,1], t \in[0,1]$,

$$
s_{r, t}\left((L, o) \sqcup\left(L^{\prime}, o^{\prime}\right)\right)=s_{r, t}(L, o)+s_{r, t}\left(L^{\prime}, o^{\prime}\right)
$$

Proof. As $(\mathbb{Z} \oplus \mathbb{Z} \oplus \mathbb{Z})$-filtered complexes,

$$
\mathcal{C}_{\text {tot }}\left(L \sqcup L^{\prime}\right)=\mathcal{C}_{\text {tot }}(L) \otimes \mathcal{C}_{\text {tot }}\left(L^{\prime}\right)
$$

Suppose first that the wrapping numbers of at least one of $L$ or $L^{\prime}$ is even. Then $g\left(o \sqcup o^{\prime}\right)=$ $g(o) \otimes g\left(o^{\prime}\right)$, and the statement of the proposition follows.

In the case that the wrapping numbers of $L$ and $L^{\prime}$ are both odd, we have that

$$
g\left(o \sqcup o^{\prime}\right)=g(o) \otimes g\left(-o^{\prime}\right)=g(-o) \otimes g\left(o^{\prime}\right) .
$$

The two tensor product expressions on the right are achieved by considering the two possible annular diagrams for $L \sqcup L^{\prime}$. (Begin with annular diagrams for the individual links, and then identify the inner boundary of one annulus with the outer boundary of the other, or vice versa.) Thus, we have

$$
s_{r, t}\left((L, o) \sqcup\left(L^{\prime}, o^{\prime}\right)\right)=s_{r, t}(L, o)+s_{r, t}\left(L^{\prime},-o^{\prime}\right)=s_{r, t}(L,-o)+s_{r, t}\left(L^{\prime}, o^{\prime}\right) .
$$

In particular, when $L^{\prime}=\widehat{\mathbb{1}}_{1}$ is the trivial 1-braid closure, an easy computation for both orientations $o^{\prime}$ and $-o^{\prime}$ on $\widehat{\mathbb{1}}_{1}$ shows that $s_{r, t}\left(\widehat{\mathbb{1}}_{1}, o^{\prime}\right)=s_{r, t}\left(\widehat{\mathbb{1}}_{1},-o^{\prime}\right)$. Thus, we have $s_{r, t}(L, o)=$ $s_{r, t}(L,-o)$ for any link with odd wrapping number. Therefore,

$$
s_{r, t}\left((L, o) \sqcup\left(L^{\prime}, o^{\prime}\right)\right)=s_{r, t}(L, o)+s_{r, t}\left(L^{\prime}, o^{\prime}\right)
$$

as desired.

Proposition 3.31. Let $(L, o) \subset A \times I$ be an oriented annular link. Then for all $r \in[0,1]$, $t \in[0,1]$,

$$
s_{r, t}(L, o)=s_{r, t}(L,-o)
$$


Proof. By Proposition 3.30, we have

$$
s_{r, t}\left((L, o) \sqcup\left(\widehat{\mathbb{1}}_{1}, o^{\prime}\right)\right)=s_{r, t}(L, o)+s_{r, t}\left(\widehat{\mathbb{1}}_{1}, o^{\prime}\right) .
$$

By considering the annular diagram for $(L, o) \sqcup\left(\widehat{\mathbb{1}}_{1}, o^{\prime}\right)$ where we identify the outer annulus of the diagram for $(L, o)$ with the inner annulus for $\left(\widehat{\mathbb{1}}_{1}, o^{\prime}\right)$, we have

$$
s_{r, t}\left((L, o) \sqcup\left(\widehat{\mathbb{1}}_{1}, o^{\prime}\right)\right)=s_{r, t}(L,-o)+s_{r, t}\left(\widehat{\mathbb{1}}_{1}, o^{\prime}\right) .
$$

The statement follows.

We study the behavior of a braid under Markov stabilizations.

Proposition 3.32. Let $\sigma \in \mathfrak{B}_{n}$ and suppose $\sigma^{ \pm} \in \mathfrak{B}_{n+1}$ is obtained from $\sigma$ by either a positive or negative Markov stabilization. Then for all $r \in[0,1], t \in[0,1]$,

$$
s_{r, t}(\widehat{\sigma})-(1-r) t \leq s_{r, t}\left(\widehat{\sigma}^{ \pm}\right) \leq s_{r, t}(\widehat{\sigma})+(1-r) t
$$

Proof. Consider the oriented annular cobordism from $\widehat{\sigma}^{ \pm}$to $\widehat{\sigma} \sqcup \widehat{\mathbb{1}}_{1}$ (the horizontal composition of $\widehat{\sigma}$ with the trivial 1-braid closure) with a single odd-index critical point that resolve the extra \pm crossing. The associated chain map on the Sarkar-Seed-Szabó complex sends $g\left(\widehat{\sigma}^{ \pm}, o_{\uparrow}\right)$ to $g\left(\widehat{\sigma} \sqcup \widehat{\mathbb{1}}_{1}, o_{\uparrow}\right)$. By Proposition 3.12, this map is filtered of degree $(1-r)(-1)$. Therefore,

$$
s_{r, t}\left(\widehat{\sigma}^{ \pm}\right)-(1-r) \leq s_{r, t}\left(\widehat{\sigma} \sqcup \widehat{\mathbb{1}}_{1}\right) .
$$

Using additivity of $s_{r, t}$ under horizontal composition (cf. Proposition 3.30) and Theorem 3.24, we have

$$
s_{r, t}\left(\widehat{\sigma} \sqcup \widehat{\mathbb{1}}_{1}\right)=s_{r, t}(\widehat{\sigma})-(1-r)(1-t)
$$

which gives us one of our two desired inequalities:

$$
s_{r, t}\left(\widehat{\sigma}^{ \pm}\right) \leq s_{r, t}(\widehat{\sigma})+(1-r) t .
$$

For the other inequality, consider the cobordism $F: \widehat{\sigma} \rightarrow \widehat{\sigma}^{ \pm}$consisting of one nonannular birth and one saddle. Then the associated filtered chain map $\phi_{F}$ increases $g r_{r, t}$ by at least $-t(1-r)$ by Proposition 3.12. Hence $s_{r, t}(\widehat{\sigma})-t(1-r) \leq s_{r, t}\left(\widehat{\sigma}^{ \pm}\right)$.

Proposition 3.33. Let $\sigma \in \mathfrak{B}_{n}$ and suppose that $\sigma^{+} \in \mathfrak{B}_{n+1}$ is obtained from $\sigma$ by performing a positive stabilization. For $t<1$ and sufficiently close to 1 ,

$$
m_{r, t}\left(\widehat{\sigma}^{+}\right)-m_{r, t}(\widehat{\sigma}) \geq 1 \text {. }
$$

Proof. Suppose $\sigma$ has writhe $w$, then $\sigma^{+}$has writhe $w+1$. We fix $r$ throughout this proof. By Theorem 3.25, we have that $s_{r, 1}(\widehat{\sigma})=(1-r) w$ and $s_{r, 1}\left(\widehat{\sigma}^{+}\right)=(1-r)(w+1)$. For the purposes of this proof, let $m=m_{r, t}(\widehat{\sigma})$ and $m^{+}=m_{r, t}\left(\widehat{\sigma}^{+}\right)$denote the right-hand slope of $s_{r, t}$ for $t<1$ and sufficiently close to 1 . Since the functions $s_{r, t}(\widehat{\sigma})$ and $s_{r, t}\left(\widehat{\sigma}^{+}\right)$are piecewise linear with respect to $t$, for $t<1$ and sufficiently close to 1 , we have that

$$
\begin{aligned}
s_{r, t}(\widehat{\sigma}) & =m t+(1-r) w-m \\
s_{r, t}\left(\widehat{\sigma}^{+}\right) & =m^{+} t+(1-r)(w+1)-m^{+}
\end{aligned}
$$

By Proposition 3.32, we have that

$$
\begin{aligned}
& s_{r, t}\left(\widehat{\sigma}^{+}\right)-s_{r, t}(\widehat{\sigma}) \leq(1-r) t \\
& \left(m^{+}-m\right)(t-1) \leq(1-r)(t-1)
\end{aligned}
$$


Thus, $m^{+}-m \geq(1-r)$. Since $m^{+}-m$ are integers of different parity mod 2 by part 4 of Theorem 3.15, the lower bound can be improved to 1 .

Inspired by the sets introduced in GLW17, Remark 15], we study the following set of braids whose $s_{r, t}(\widehat{\sigma})$ invariants attain the maximal slope for some $t_{0} \in[0,1)$, and therefore, by Proposition 3.29 for all $t \in\left[t_{0}, 1\right)$.

Definition 3.34. Let $t_{0} \in[0,1)$. Define

$$
\mathfrak{M}_{t_{0}}^{\prime}=\left\{\text { Braids } \sigma \mid m_{r, t}=(1-r) n \text { for all } t \in\left[t_{0}, 1\right) \text { and all } r \in[0,1]\right\} .
$$

The following two lemmas show that membership in $\mathfrak{M}_{t_{0}}^{\prime}$ is preserved under positive stabilization.

Lemma 3.35. Let $\sigma \in \mathfrak{B}_{n}$ have writhe $w$, and suppose $\sigma^{\prime} \in \mathfrak{B}_{n}$ is obtained from $\sigma$ by inserting a single positive crossing. Then if $\sigma \in \mathfrak{M}_{t_{0}}^{\prime}$ for some $t_{0} \in[0,1)$, then $\sigma^{\prime} \in \mathfrak{M}_{t_{0}}^{\prime}$.

Proof. Since $\sigma \in \mathfrak{M}_{t_{0}}^{\prime}$, we know that for each $t \in\left[t_{0}, 1\right)$, we have

$$
s_{r, t}(\widehat{\sigma})=g r_{r, t}\left(\mathbf{v}_{-}(\widehat{\sigma})\right)=(1-r)((-n+w)+n t) .
$$

Note that $\sigma^{\prime}$ has writhe $w+1$. By applying part 5 of Theorem 3.15 to the annular saddle cobordism $\widehat{\sigma}^{\prime} \rightarrow \widehat{\sigma}$ that resolves the single extra positive crossing tells us

$$
s_{r, t}\left(\widehat{\sigma}^{\prime}\right)-s_{r, t}(\widehat{\sigma}) \leq(1-r)
$$

and hence,

$$
s_{r, t}\left(\widehat{\sigma}^{\prime}\right) \leq(1-r)((-n+w+1)+n t)
$$

On the other hand,

$$
(1-r)((-n+w+1)+n t)=g r_{r, t}\left(g\left(\widehat{\sigma}^{\prime}\right)\right) \leq s_{r, t}\left(\widehat{\sigma}^{\prime}\right) .
$$

Hence $m_{r, t}\left(\widehat{\sigma}^{\prime}\right)=(1-r) n$ for all $t \in\left[t_{0}, 1\right)$ as desired.

Lemma 3.36. Let $t_{0} \in[0,1)$. Let $\sigma \in \mathfrak{B}_{n}$ and $\sigma^{\prime} \in \mathfrak{B}_{n^{\prime}}$, and let $\sigma \sqcup \sigma^{\prime} \in \mathfrak{B}_{n+n^{\prime}}$ denote their horizontal composition. If $\sigma, \sigma^{\prime} \in \mathfrak{M}_{t_{0}}^{\prime}$, then $\sigma \sqcup \widehat{\sigma}^{\prime} \in \mathfrak{M}_{t_{0}}^{\prime}$.

Proof. This follows immediately from Proposition 3.30 .

The behavior of the $s_{r, t}$ invariant under positive destabilization is currently unknown. As described in the introduction, a complete understanding of the behavior of $s_{r, t}$ under positive stabilization and destabilization could potentially yield a new transverse invariant. This invariant may or may not be effective, or contain more information than the self-linking number. The question of whether Plamenevskaya's transverse link invariant $\psi$ [Pla06] is effective remains open. It is shown in [BP10, Theorem 1.2] that for braids representing Khovanov thin knot types, the vanishing of the transverse link invariant $\psi$ only depends on the $s$-invariant and the self-linking number. Recently, Martin [Mar] used $d_{t}$ to show that for 3-braid closures, the vanishing of Plamenvskaya's transverse link invariant $\psi$ depends only on the $s$-invariant and and the self-linking number. In this direction, it is natural to ask whether the $s_{r, t}$ invariant of a braid closure only depends on $s_{\mathbb{F}_{2}}$ and the self-linking number.

In another direction, we produce a lower bound on the band rank $\mathrm{rk}_{n}$ of a braid [Rud83]. Given $\beta \in \mathfrak{B}_{n}$,

$$
\operatorname{rk}_{n}(\beta):=\min \left\{c \in \mathbb{Z}^{\geq 0} \mid \beta=\prod_{j=1}^{c} \omega_{j} \sigma_{i_{j}}^{ \pm}\left(\omega_{j}\right)^{-1} \text { for some } \omega_{j} \in \mathfrak{B}_{n}\right\}
$$


where $\sigma_{i_{j}}$ denotes the elementary Artin generators. Note that band rank $\mathrm{rk}_{n}$ is a braid conjugacy class invariant. Topologically, band $\operatorname{rank}_{n}(\beta)$ is the minimum number of halftwist bands (running perpendicularly to the strands) needed to construct a Seifert surface for $\widehat{\beta}$ from $n$ disks (the obvious Seifert surface for the identity braid closure in $\mathfrak{B}_{n}$ ).

Proposition 3.37. Let $r \neq 1$. Given an oriented cobordism $F$ from $(L, o)$ to $\left(L^{\prime}, o^{\prime}\right)$ with $a_{0}$ annular even index critical points, $a_{1}$ annular odd index critical points, and $b_{0}$ non-annular even index critical points, we have

$$
\left|\frac{s_{r, t}(\hat{\beta})}{1-r}+n(1-t)\right| \leq r k_{n}(\beta) .
$$

Proof. Recall $\mathbb{1}_{n}$ denotes the identity braid in $B_{n}$. Observe that $s_{r, t}\left(\widehat{\mathbb{1}}_{n}\right)=\operatorname{gr}_{r, t}\left(v_{-} \otimes v_{-} \otimes\right.$ $\left.\cdots \otimes v_{-}\right)=(1-r)(-n+t n)=-n(1-r)(1-t)$.

Let $F$ be the cobordism from $\widehat{\beta}$ to $\widehat{\mathbb{1}}_{n}$ realizing the band rank of $\widehat{\beta}$, i.e. $a_{1}=\operatorname{rk}_{n}(\beta)$. Then by Proposition 3.12 ,

$$
s_{r, t}(\widehat{\beta})-s_{r, t}\left(\widehat{\mathbb{1}}_{n}\right)=s_{r, t}(\widehat{\beta})+n(1-r)(1-t) \leq(1-r) \mathrm{rk}_{n}(\beta) .
$$

Since $-F$ is a cobordism from $\widehat{\mathbb{1}}_{n}$ to $\widehat{\beta}$ with the same $a_{1}$, we have $\left|s_{r, t}(\widehat{\beta})-s_{r, t}\left(\widehat{\mathbb{1}}_{n}\right)\right| \leq$ $(1-r) \mathrm{rk}_{n}(\beta)$. Finally, we divide both sides of the inequality by $1-r>0$.

\section{Functoriality}

4.1. Strong functoriality. In [Sal17], Saltz defines strong Khovanov-Floer theories and proves that any conic strong Khovanov-Floer theory is functorial.

To motivate the definition of a strong Khovanov-Floer theory, we briefly discuss what functoriality should mean, and how we will use it.

An assignment $\mathcal{K}:$ Diag $\rightarrow$ Filt (see Definition 2.4) is a functor if the following conditions are met. First of all, $\mathcal{K}$ must give a well-defined map on objects. So, if $\mathcal{K}$ describes how to map a link diagram $\mathcal{D}$ to a filtered chain complex $\mathcal{C}$, it must map all equivalent link diagrams to the chain homotopy equivalence class of $\mathcal{C}$ as well. Furthermore, oftentimes $\mathcal{K}$ will be described using some auxiliary information attached to $\mathcal{D}$; we much also check that ultimately, the assignment $\mathcal{K}(\mathcal{D})$ did not depend on this auxiliary information. Next, $\mathcal{K}$ must also give a well-defined map on morphisms. Again, it must send two equivalent diagrammatic cobordisms to homotopic maps between filtered complexes. This assignment must also not depend on any auxiliary information used to describe the assignment.

The above ensures that the assignment $\mathcal{K}$ is a functor. In the special case when $\mathcal{K}$ resembles the Khovanov homology functor, some of the above functorial properties will follow automatically. Such a functor $\mathcal{K}$ behaves as a categorification of some skein relations, and should satisfy:

- $\mathcal{K}$ agrees with $K h$ on the unknot diagram with no crossings. In terms of morphisms, this should have the structure of a Frobenius algebra with multiplication and comultiplication maps.

- $\mathcal{K}$ sends disjoint unions to tensor products.

- $\mathcal{K}$ is built from a cube of resolutions picture. This property is called conicity.

These special structural constraints help prove the functoriality of $\mathcal{K}$. For example, if $\mathcal{K}$ is conic (in Saltz's vocabulary, because it is built with mapping cones), $\mathcal{K}$ is essentially a lift of a functor from the cube category, and these are very nice diagrams to understand. For 
example, handleswap invariance follows from the fact that faces commute in many of these cubical complexes.

Once such a functor $\mathcal{K}$ is established, we can consider well-defined homology classes in $\mathcal{K}(L)$ as link invariants, and study their properties under cobordism. In particular, we may study the filtration gradings of some distinguished homology classes as in this paper.

With all this in mind, below is Saltz's definition of a strong Khovanov-Floer theory. If it seems abstract, we recommend cross-referencing with the proof of Proposition 4.5 in Section 4.2 for the concrete application needed for our results.

Definition 4.1 (Definition 3.8, Sal17]). A strong Khovanov-Floer theory is an assignment

$\mathcal{K}:\{$ Link diagrams with auxiliary data $\} \rightarrow\{$ filtered chain complexes $\}$

satisfying the following conditions:

(S-1) For two collections $A_{\alpha}$ and $A_{\beta}$ of auxiliary data associated to a diagram $\mathcal{D}$, there is a chain homotopy equivalence

$$
a_{\alpha}^{\beta}: \mathcal{K}\left(\mathcal{D}, A_{\alpha}\right) \rightarrow \mathcal{K}\left(\mathcal{D}, A_{\beta}\right)
$$

such that the collections $\left\{\mathcal{K}\left(\mathcal{D}, A_{\alpha}\right)\right\}$ together with the maps $\left\{a_{\alpha}^{\beta}\right\}$ form a transitive system in Filt. Let $\mathcal{K}(\mathcal{D})$ denote the inverse limit, or the canonical representative for this diagram $\mathcal{D}$.

(S-2) For a crossingless diagram of the unknot $\mathcal{D}$, we have $H_{\text {tot }}(\mathcal{K}(\mathcal{D})) \cong K h(\mathcal{D})$.

(S-3) For a disjoint union of diagrams $\mathcal{D} \cup \mathcal{D}^{\prime}$, we have a chain homotopy equivalence $\mathcal{K}\left(\mathcal{D} \cup \mathcal{D}^{\prime}\right) \simeq \mathcal{K}(\mathcal{D}) \otimes_{\mathbb{F}} \mathcal{K}\left(\mathcal{D}^{\prime}\right)$

(S-4) If $\mathcal{D}^{\prime}$ is the result of a diagrammatic handle attachment to $\mathcal{D}$,

- there is a function $\phi: \operatorname{Aux}(\mathcal{D}) \rightarrow \operatorname{Aux}\left(\mathcal{D}^{\prime}\right)$,

- there is a map $h_{A_{\alpha}, \phi\left(A_{\alpha}\right), B}: \mathcal{K}\left(\mathcal{D}, A_{\alpha}\right) \rightarrow \mathcal{K}\left(\mathcal{D}^{\prime}, \phi\left(A_{\alpha}\right)\right)$ where $B$ is additional auxiliary data for the handle attachment,

- for fixed $B$, these maps extend to maps on transitive systems, therefore defining a map $h_{B}: \mathcal{K}(\mathcal{D}) \rightarrow \mathcal{K}\left(\mathcal{D}^{\prime}\right)$ on canonical representatives,

- and for any two sets of additional auxiliary data $B$ and $B^{\prime}$, we have $h_{B} \simeq h_{B^{\prime}}$.

(S-5) For $U$ a crossingless diagram of an unknot, $\mathcal{K}(U)$ is a Frobenius algebra $\mathcal{A}$ with the operations $\iota: \mathbb{F} \rightarrow \mathcal{A}, \epsilon: \mathcal{A} \rightarrow \mathbb{F}, m: \mathcal{A} \otimes \mathcal{A} \rightarrow \mathcal{A}$, and $\Delta: \mathcal{A} \rightarrow \mathcal{A} \otimes \mathcal{A}$ given by diagrammatic birth, death, merge, and split, respectively.

(S-6) If $\mathcal{D}^{\prime}$ is the result of a planar isotopy on $\mathcal{D}$, then $\mathcal{K}(\mathcal{D}) \simeq \mathcal{K}\left(\mathcal{D}^{\prime}\right)$.

(S-7) If $\mathcal{D}=\mathcal{D}_{0} \sqcup \mathcal{D}_{1}, \mathcal{D}^{\prime}=\mathcal{D}_{0}^{\prime} \sqcup \mathcal{D}_{1}^{\prime}$, and $\Sigma$ is a diagrammatic cobordism $\mathcal{D} \rightarrow \mathcal{D}^{\prime}$ that is a disjoint union of cobordisms $\Sigma_{0}: \mathcal{D}_{0} \rightarrow \mathcal{D}_{0}^{\prime}$ and $\Sigma_{1}: \mathcal{D}_{1} \rightarrow \mathcal{D}_{1}^{\prime}$, then $\mathcal{K}(\Sigma) \simeq \mathcal{K}\left(\Sigma_{0}\right) \otimes \mathcal{K}\left(\Sigma_{1}\right)$.

(S-8) The handle attachment maps satisfy handleswap invariance and movie move 15, up to homotopy.

Definition 4.2 (Definition 3.9, SSal17]). Let $c$ be some crossing in a link diagram $\mathcal{D}$. For $i=0,1$, let $\mathcal{D}_{i}$ be the diagram where $c$ is replaced by its $i$-resolution. Let $\gamma$ be the arc in $\mathcal{D}_{0}$ along which a 1-handle is attached to produce the diagram $\mathcal{D}_{1}$. A strong Khovanov-Floer theory $\mathcal{K}$ is conic if $\mathcal{K}(\mathcal{D}) \simeq \operatorname{cone}\left(h_{\gamma}: \mathcal{K}\left(\mathcal{D}_{0}\right) \rightarrow \mathcal{K}\left(\mathcal{D}_{1}\right)\right)$ where $h_{\gamma}$ is the handle attachment map.

Theorem 4.3 ([Sal17]). Conic, strong Khovanov-Floer theories are functorial. In other words, $\mathcal{K}$ gives rise to a functor Diag $\rightarrow \operatorname{Kom}($ Vect $)$, the homotopy category of complexes over $\mathbb{F}_{2}$. 


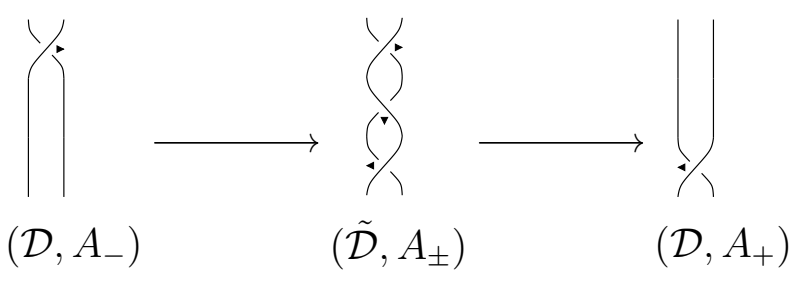

Figure 8. Changing the decoration at a single crossing.

Remark 4.4. Saltz dubbed this functoriality property "strong" to differentiate from the functoriality of Khovanov-Floer spectral sequences described by Baldwin-Hedden-Lobb in [BHL19]. The key difference is that even though under some finiteness conditions $E^{\infty}$ is isomorphic to the total homology of a filtered complex, the map induced between $E^{\infty}$ pages does not always agree with the map induced on homology.

4.2. Functoriality of the Sarkar-Seed-Szabó complex. In [Sal17, Theorem 6.9], Saltz proves that Szabó's geometric spectral sequence is a functorial link invariant. Here we prove that the Sarkar-Seed-Szabó complex is a functorial link invariant.

Proposition 4.5. The Sarkar-Seed-Szabó theory $\mathcal{K}(\mathcal{D})=\left(\mathcal{C}_{\text {ftot }}, \partial_{\text {ftot }}\right)$ is a conic, strong Khovanov-Floer theory, and is therefore functorial.

Proof. First of all, conicity follows by Sarkar-Seed-Szabó's definition of saddle maps [SSS17, Definition 5.2].

(S-1) Suppose for a diagram $\mathcal{D}$ two sets of decorations $A_{-}$and $A_{+}$differ at exactly one crossing $c$; here we think of - and + as the two ways to decorate the crossing $c$. We form a diagram $\tilde{\mathcal{D}}$ differing from $\mathcal{D}$ in a small disk around $c$ that replaces $c$ with three crossings, by way of a Reidemeister II move, as in Figure 8. We associate a decoration $A_{ \pm}$to $\tilde{\mathcal{D}}$ as follows: the two outer crossings are labeled with opposite decoration choices, labeled - and + , and we choose an arbitrary decoration for the middle crossing. By [SSS17, Corollary 4.3], the complex $\mathcal{K}\left(\tilde{\mathcal{D}}, A_{ \pm}\right)$is chain homotopy equivalent to each of $\mathcal{K}\left(\mathcal{D}, A_{-}\right)$and $\mathcal{K}\left(\mathcal{D}, A_{+}\right)$; Sarkar-Seed-Szabó use the cancellation lemma to show that these chain isomorphisms are cancellations of acyclic direct summands. (A change of basis allows the complex to split.) The chain homotopy equivalence $a_{-}^{+}: \mathcal{K}\left(\mathcal{D}, A_{-}\right) \rightarrow \mathcal{K}\left(\mathcal{D}, A_{+}\right)$is the composition

$$
a_{-}^{+}=\widetilde{a}_{ \pm}^{+} \circ \widetilde{b}_{-}^{ \pm}
$$

where $\widetilde{a}_{ \pm}^{-}: \mathcal{K}\left(\tilde{\mathcal{D}}, A_{ \pm}\right) \rightarrow \mathcal{K}\left(\mathcal{D}, A_{-}\right)$and $\widetilde{a}_{ \pm}^{+}: \mathcal{K}\left(\tilde{\mathcal{D}}, A_{ \pm}\right) \rightarrow \mathcal{K}\left(\mathcal{D}, A_{+}\right)$are the SarkarSeed-Szabó Reidemeister II chain homotopy equivalences described in Proposition 4.7 of [SSS17], and $\widetilde{b}_{-}^{ \pm}: \mathcal{K}\left(\mathcal{D}, A_{-}\right) \rightarrow \mathcal{K}\left(\tilde{\mathcal{D}}, A_{ \pm}\right)$is the chain map induced by the identification of $\left(\mathcal{D}, A_{-}\right)$with the "01" partial resolution of $\left(\tilde{\mathcal{D}}, A_{ \pm}\right)$and satisfies $\widetilde{a}_{ \pm}^{-} \circ \widetilde{b}_{-}^{ \pm} \simeq \mathrm{id}$.

Now for decorations $A_{\alpha}$ and $A_{\beta}$ on $\mathcal{D}$ differing at multiple crossings, define the map relating their complexes

$$
a_{\alpha}^{\beta}: \mathcal{K}\left(\mathcal{D}, A_{\alpha}\right) \rightarrow \mathcal{K}\left(\mathcal{D}, A_{\beta}\right)
$$


as a composition of the chain homotopy equivalences of single crossing changes. If this is well-defined (i.e. does not depend on the order of crossings we choose to compose the chain homotopy equivalences), then these maps form a transitive system.

Thus, without loss of generality, we may assume that the decorations $A_{\alpha}=A_{-,-}$ and $A_{\beta}=A_{+,+}$differ at exactly two crossings, $c_{1}$ and $c_{2}$. Let $A_{\eta^{\prime}}=A_{+,-}$and $A_{\eta}=A_{-,+}$be the two decorations for $\mathcal{D}$ that differ from $A_{\alpha}$ by exactly one decoration change, at either $c_{1}$ or $c_{2}$, respectively. To show that the system of crossing change maps is transitive, we need to check that

$$
a_{\eta^{\prime}}^{\beta} \circ a_{\alpha}^{\eta^{\prime}} \simeq a_{\eta}^{\beta} \circ a_{\alpha}^{\eta} .
$$

By decoration invariance of the Sarkar-Seed-Szabó complex, $\mathcal{K}\left(\tilde{\tilde{\mathcal{D}}}, A_{ \pm, \pm}\right)$is chain homotopy equivalent to each chain complex $\mathcal{K}\left(\mathcal{D}, A_{\alpha}\right), \mathcal{K}\left(\mathcal{D}, A_{\beta}\right), \mathcal{K}\left(\mathcal{D}, A_{\eta}\right)$, and $\mathcal{K}\left(\mathcal{D}, A_{\eta^{\prime}}\right)$. By symmetry, it suffices to show that the box below commutes up to homotopy (the diagram names are omitted but should be clear from the decoration notation):

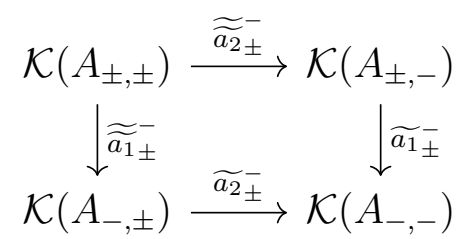

where the chain isomorphism subscripts, 1 or 2 , indicate the crossing, $c_{1}$ or $c_{2}$, associated to the Reidemeister II chain map. A detailed description of each composition requires drawing the 4-dimensional cube of $2^{4}$ vertices in the partial cube of resolutions corresponding to the four crossings we wish to eliminate, and performing cancellations according to the usual proof (described in Proposition 4.7 of [SSS17]), twice, as illustrated in Figure 9. Ultimately, we find that both chain homotopy equivalences identify $\mathcal{K}\left(A_{-,-}\right)$with the complex $\mathcal{K}\left(A_{ \pm, \pm}\right)_{(1,0,1,0)}$, situated at the vertex $(1,0,1,0)$ of this 4-dimensional cube.

(S-2) This is clear by definition at the chain level. Since there are no differentials, this also holds in homology.

(S-3) This holds at the chain level by definition. Furthermore, there are no cross-differentials (i.e. differentials are of the form $\phi \otimes \psi$ ) between the two tensor factors, so this also holds for homology.

$(\mathrm{S}-4)$ Let $\mathcal{D}$ and $\mathcal{D}^{\prime}$ differ by a diagrammatic handle-attachement, and let $\operatorname{Aux}(\mathcal{D})$ denote the set of decorations for the diagram $\mathcal{D}$. There is a canonical bijection $\phi: \operatorname{Aux}(\mathcal{D}) \rightarrow$ $\operatorname{Aux}\left(\mathcal{D}^{\prime}\right)$, in the sense that $\mathcal{D}$ and $\mathcal{D}^{\prime}$ differ only on a disk on which there are no crossings to decorate. For a 0- or 2-handle attachment, there is no additional auxiliary data $B$ for the cobordism. For a 1-handle attachment, the auxiliary data $B$ is consists of the oriented arc $\gamma$ along which surgery is performed. Let $-\gamma$ denote the oppositely oriented arc.

Let $A_{\alpha}$ and $A_{\beta}$ denote decorations for a diagram $\mathcal{D}$. Let $\mathcal{D}_{\gamma}$ be the diagram obtained from $\mathcal{D}$ by adding an extra crossing as follows: the directed arc $\gamma$ is replaced with a crossing whose 0 -resolution is $\mathcal{D}_{\gamma ; 0}$ and whose 1 -resolution is $\mathcal{D}_{\gamma ; 1}$, decorated to agree with the orientation of $\gamma$. Define $\mathcal{D}_{-\gamma}$ similarly; the only difference is the decoration at the new crossing. Thus, $\mathcal{D}=\mathcal{D}_{\gamma ; 0}$ and $\mathcal{D}^{\prime}=\mathcal{D}_{\gamma ; 1}$ differ by a diagrammatic onehandle attachment. Associated to the additional auxiliary data of the oriented arc 

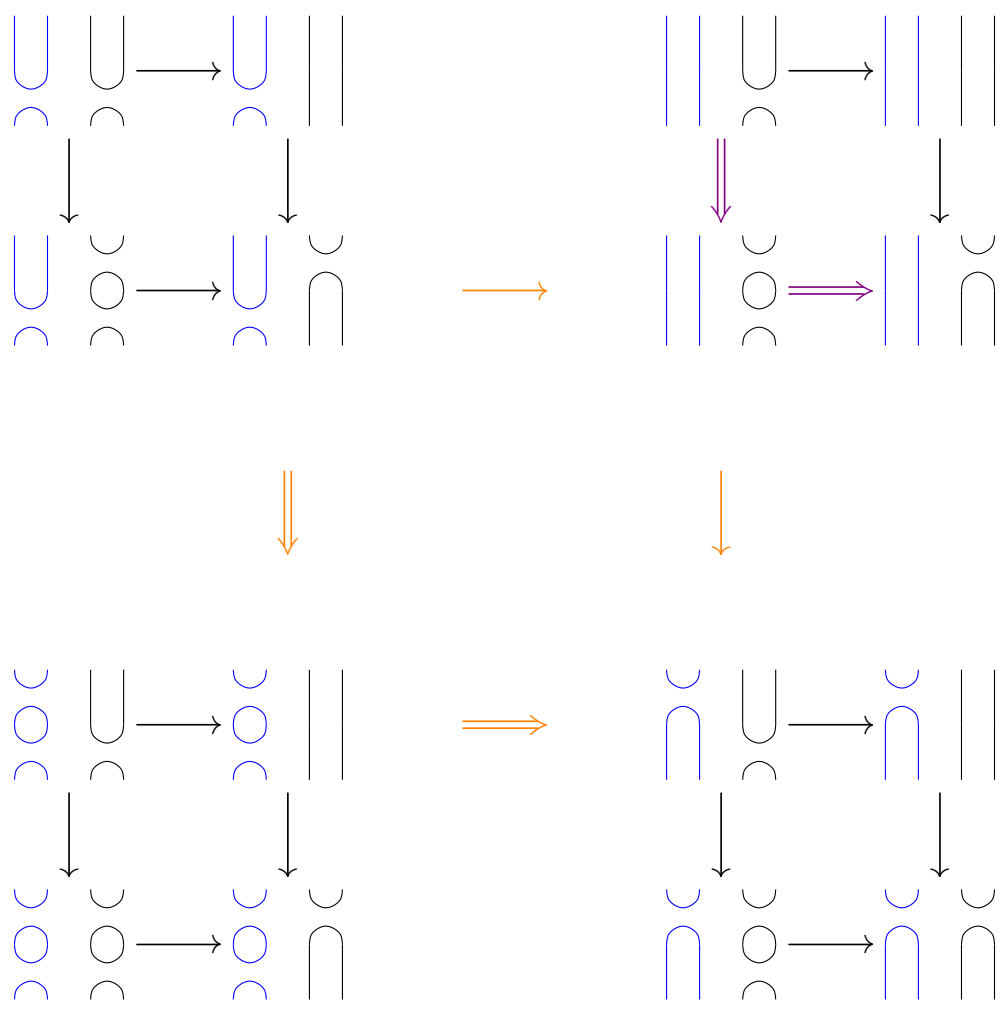

Figure 9. The partial cube of resolutions of $\widetilde{\widetilde{\mathcal{D}}}$ for the RII invariance. We first cancel along the orange double arrows, then along the violet double arrows between the " $01 * *$ " resolutions, leaving a subquotient complex isomorphic to the complex for $D$.

$\gamma$, there are chain homotopy equivalences $a_{\alpha ; 0}^{\beta}$ and $a_{\alpha ; 1}^{\beta}$ as defined in (S-1), and there exist chain maps $f_{\alpha, \gamma}$ and $f_{\beta, \gamma}$ as in the following diagram:

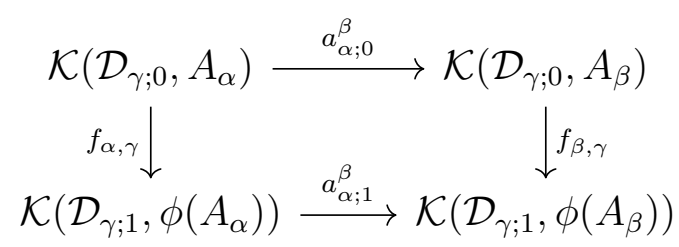

We will show the above diagram commutes up to homotopy. Observe that the left and right columns are the mapping cone complexes cone $\left(f_{\alpha, \gamma}\right) \simeq \mathcal{K}\left(\mathcal{D}_{\gamma}, A_{\alpha}\right)$ and cone $\left(f_{\beta, \gamma}\right) \simeq \mathcal{K}\left(\mathcal{D}_{\gamma}, A_{\beta}\right)$, respectively. Here we abuse notation by using $A_{\alpha}$ to denote the decoration on $\mathcal{D}_{\gamma}$ associated to adding the decoration induced by $\gamma$ on the additional crossing. By decoration invariance of the Sarkar-Seed-Szabó complex up to chain homotopy, as in (S-1) we have a chain homotopy equivalence $a_{\alpha}^{\beta}: \mathcal{K}\left(\mathcal{D}_{\gamma}, A_{\alpha}\right) \rightarrow \mathcal{K}\left(\mathcal{D}_{\gamma}, A_{\beta}\right)$. 
Claim 4.6. As a chain homotopy equivalence between mapping cones, the chain map $a_{\alpha}^{\beta}: \mathcal{K}\left(\mathcal{D}_{\gamma}, A_{\alpha}\right) \rightarrow \mathcal{K}\left(\mathcal{D}_{\gamma}, A_{\beta}\right)$ is of the form $\left[\begin{array}{cc}a_{\alpha ; 0}^{\beta} & a_{10} \\ a_{01} & a_{\alpha ; 1}^{\beta}\end{array}\right]$.

Proof. Write $a_{\alpha}^{\beta}=\left[\begin{array}{ll}a_{00} & a_{10} \\ a_{01} & a_{11}\end{array}\right]$ and consider the diagonal entries. The decoration changes between $A_{\alpha}$ and $A_{\beta}$ occur at only crossings that do not include the crossing associated to $\gamma$. Thus, the (Reidemeister II invariance) cancellation data used to define $a_{\alpha}^{\beta}$ are the union of the arrows used to define $a_{\alpha ; 0}^{\beta}$ and $a_{\alpha ; 1}^{\beta}$. Any zigzag differentials induced by these cancellations must travel between resolutions where crossing $\gamma$ is 0 -resolved to resolutions where crossing $\gamma$ is 1-resolved, and therefore do not affect either of the diagonal entries.

In particular, $a_{\alpha}^{\beta}=\left[\begin{array}{cc}a_{\alpha ; 0}^{\beta} & a_{10} \\ a_{01} & a_{\alpha ; 1}^{\beta}\end{array}\right]$ is a chain map, where $a_{01}: \mathcal{K}\left(\mathcal{D}_{\gamma ; 0}, A_{\alpha}\right) \rightarrow$ $\mathcal{K}\left(\mathcal{D}_{\gamma ; 1}, \phi\left(A_{\beta}\right)\right)$. Thus, $a_{\alpha}^{\beta} \circ \partial_{\mathcal{D}_{\gamma, \alpha}}+\partial_{\mathcal{D}_{\gamma, \beta}} \circ a_{\alpha}^{\beta}=0$, or

$$
\left[\begin{array}{cc}
a_{\alpha ; 0}^{\beta} & a_{10} \\
a_{01} & a_{\alpha ; 1}^{\beta}
\end{array}\right]\left[\begin{array}{cc}
\partial_{\gamma ; 0}^{\alpha} & 0 \\
f_{\alpha, \gamma} & \partial_{\gamma ; 1}^{\alpha}
\end{array}\right]+\left[\begin{array}{cc}
\partial_{\gamma ; 0}^{\beta} & 0 \\
f_{\beta, \gamma} & \partial_{\gamma ; 1}^{\beta}
\end{array}\right]\left[\begin{array}{cc}
a_{\alpha ; 0}^{\beta} & a_{10} \\
a_{01} & a_{\alpha ; 1}^{\beta}
\end{array}\right]=0 .
$$

The bottom left entry of the resulting matrix shows that the square commutes up to homotopy, via the homotopy $a_{01}$.

Then, by Lemma 3.6 of [Sal17], the maps $f_{A_{\alpha}, \phi\left(A_{\alpha}\right), \gamma}$ and $f_{A_{\beta}, \phi\left(A_{\beta}\right), \gamma}$ can be extended to a map $f_{\gamma}$ on the canonical representative of $\mathcal{K}(\mathcal{D})$. Hence we may write $f_{\gamma}$ (and $\left.f_{-\gamma}\right)$ as a map $\mathcal{K}\left(\mathcal{D}_{\gamma ; 0}\right) \rightarrow \mathcal{K}\left(\mathcal{D}_{\gamma ; 1}\right)$, and it remains to show that $f_{\gamma} \simeq f_{-\gamma}$.

We write $H_{\gamma}=\left[\begin{array}{cc}0 & 0 \\ h_{\{\gamma\}} & 0\end{array}\right]$ for the change of decoration isomorphism at the crossing corresponding to $\gamma($ and $-\gamma)$, where $h_{\{\gamma\}}: \mathcal{K}\left(\mathcal{D}_{\gamma ; 0}\right) \rightarrow \mathcal{K}\left(\mathcal{D}_{\gamma ; 1}\right)$ is the map in Definition 3.6 of [SSS17]. We will first show that

$$
\delta_{f t o t, \gamma}+\delta_{f t o t,-\gamma}=H_{\gamma} \circ \delta_{f t o t, \gamma}+\delta_{f t o t, \gamma} \circ H_{\gamma}
$$

Recall $\delta_{\text {ftot }, \gamma}=\mathbf{d}_{\mathcal{D}_{\gamma}}+\mathbf{h}_{\mathcal{D}_{\gamma}}$, where $\mathbf{d}_{\mathcal{D}_{\gamma}}=\sum_{i} d_{i, \mathcal{D}_{\gamma}}$ and $\mathbf{h}_{\mathcal{D}_{\gamma}}=\sum_{i} h_{i, \mathcal{D}_{\gamma}}$. By the proof of Lemma 3.10 [SSS17],

$$
\mathbf{d}_{\mathcal{D}_{\gamma}}+\mathbf{d}_{\mathcal{D}_{-\gamma}}=H_{\gamma} \circ \mathbf{d}_{\mathcal{D}_{\gamma}}+\mathbf{d}_{\mathcal{D}_{\gamma}} \circ H_{\gamma}
$$

By Corollary 3.9 of [SSS17], $H_{\gamma}$ commutes with $\mathbf{h}_{\mathcal{D}_{\gamma}}$. Moreover, the map $\mathbf{h}_{\mathcal{D}_{\gamma}}$ is independent of the choice of orientation of $\gamma$, and hence, $\mathbf{h}_{\mathcal{D}_{\gamma}}=\mathbf{h}_{\mathcal{D}_{-\gamma}}$. Thus,

$$
\delta_{f t o t, \gamma}+\delta_{f t o t,-\gamma}=\mathbf{d}_{\mathcal{D}_{\gamma}}+\mathbf{h}_{\mathcal{D}_{\gamma}}+\mathbf{d}_{\mathcal{D}_{-\gamma}}+\mathbf{h}_{\mathcal{D}_{-\gamma}}=H_{\gamma} \circ \delta_{f t o t, \gamma}+\delta_{f t o t, \gamma} \circ H_{\gamma} .
$$

This immediately implies that

$$
f_{\gamma}+f_{-\gamma}=h_{\{\gamma\}} \circ \delta_{f t o t, \gamma ; 0}+\delta_{f t o t,-\gamma ; 1} \circ h_{\{\gamma\}}
$$

hence $f_{\gamma} \simeq f_{-\gamma}$.

(S-5) The Frobenius algebra is $\mathbb{E}[x] /(x(x+1))$, since the differential for a diagram with only one crossing is just $d_{1}+h_{1}$ which is the Bar-Natan differential.

(S-6) This condition follows from the proof that the Sarkar-Seed-Szabó theory $\mathcal{C}_{\text {tot }}$ (and thus, $\mathcal{C}_{\text {ftot }}$ ) is invariant under Reidemeister moves. See Corollary 4.3 of [SSS17]. 
(S-7) Resolution configurations that don't share any active circles won't give cross differentials between the two tensor factors. In the language of Sarkar-Seed-Szabó the differentials satisfy the extension rule (see [SSS17, Lemma 3.3]. We can view the disjoint union cobordism $\Sigma=\Sigma_{0} \sqcup \Sigma_{1}$ as a composition of cobordisms $\left.\Sigma_{0} \sqcup \operatorname{id}_{\mathcal{D}_{1}}\right)$ and $\left.\operatorname{id}_{\mathcal{D}_{0}^{\prime}} \sqcup \Sigma_{1}\right)$. By the extension rule, we have

$$
\mathcal{K}(\Sigma) \simeq\left(\operatorname{id}_{\mathcal{D}_{0}^{\prime}} \otimes \mathcal{K}\left(\Sigma_{1}\right)\right) \circ\left(\mathcal{K}\left(\Sigma_{0}\right) \otimes \operatorname{id}_{\mathcal{D}_{1}}\right)=\mathcal{K}\left(\Sigma_{0}\right) \otimes \mathcal{K}\left(\Sigma_{1}\right)
$$

(S-8) Movie move 15 invariance follows from conicity and Proposition 6.2 of [Sal17]. One can also check this directly, by comparing the identity map to a birth followed by a merge; dually, compare the identity map to a split followed by a death. (Recall that the birth map sends $x \mapsto x \otimes v_{+}$.)

Handleswap invariance follows from conicity and Proposition 6.1 of [Sal17]. Both of the cobordism maps $h_{\gamma^{\prime}} \circ h_{\gamma}$ and $h_{\gamma} \circ h_{\gamma^{\prime}}$ are given by the sum of all the differentials in the complex $\mathcal{D}_{\gamma \gamma^{\prime}}=\mathcal{D}_{\gamma^{\prime} \gamma}$ from the 00-resolution to the 11-resolution.

\section{REFERENCES}

[BHL19] John A. Baldwin, Matthew Hedden, and Andrew Lobb. On the functoriality of Khovanov-Floer theories. Adv. Math., 345:1162-1205, 2019.

[BN02] Dror Bar-Natan. On Khovanov's categorification of the Jones polynomial. Algebr. Geom. Topol., 2:337-370, 2002.

[BN05] Dror Bar-Natan. Khovanov's homology for tangles and cobordisms. Geom. Topol., 9:1443-1499, 2005.

[BP10] John A. Baldwin and Olga Plamenevskaya. Khovanov homology, open books, and tight contact structures. Adv. Math., 224(6):2544-2582, 2010.

[CS93] J. Scott Carter and Masahico Saito. Reidemeister moves for surface isotopies and their interpretation as moves to movies. J. Knot Theory Ramifications, 2(3):251-284, 1993.

[DHST18] Irving Dai, Jennifer Hom, Matthew Stoffregen, and Linh Truong. More concordance homomorphisms from knot Floer homology, 2018. in preparation.

[GLW17] J. Elisenda Grigsby, Anthony M. Licata, and Stephan M. Wehrli. Annular Khovanov-Lee homology, braids, and cobordisms. Pure Appl. Math. Q., 13(3):389-436, 2017.

[GLW18] J. Elisenda Grigsby, Anthony M. Licata, and Stephan M. Wehrli. Annular Khovanov homology and knotted Schur-Weyl representations. Compos. Math., 154(3):459-502, 2018.

[Gri18] J. Elisenda Grigsby. On braided, banded surfaces and ribbon obstructions, 2018. preprint, arXiv:1801.07158.

[He18] Dongtai He. Upsilon invariant, fibered knots and right-veering open books. PhD thesis, Boston College, 2018.

[HM17] Kristen Hendricks and Ciprian Manolescu. Involutive Heegaard Floer homology. Duke Math. J., 166(7):1211-1299, 2017.

[HS16] Diana Hubbard and Adam Saltz. An annular refinement of the transverse element in Khovanov homology. Algebr. Geom. Topol., 16(4):2305-2324, 2016.

[Kho00] Mikhail Khovanov. A categorification of the Jones polynomial. Duke Math. J., 101(3):359-426, 2000 .

[Lee05] Eun Soo Lee. An endomorphism of the Khovanov invariant. Adv. Math., 197(2):554-586, 2005.

[Lin19] Francesco Lin. Bar-Natan's deformation of Khovanov homology and involutive monopole Floer homology. Math. Ann., 373(1-2):489-516, 2019.

[LL19] Lukas Lewark and Andrew Lobb. Upsilon-like concordance invariants from $\mathfrak{s l}_{n}$ knot cohomology. Geom. Topol., 23(2):745-780, 2019.

[LS14] Robert Lipshitz and Sucharit Sarkar. A refinement of Rasmussen's S-invariant. Duke Math. J., 163(5):923-952, 2014.

[Mar] Gage Martin. Annular rasmussen invariants: properties and 3-braid classification. In preparation. 
[MTV07] Marco Mackaay, Paul Turner, and Pedro Vaz. A remark on Rasmussen's invariant of knots. $J$. Knot Theory Ramifications, 16(3):333-344, 2007.

[OS03] Peter Ozsváth and Zoltán Szabó. Knot Floer homology and the four-ball genus. Geom. Topol., 7:615-639, 2003.

[OS04] Peter Ozsváth and Zoltán Szabó. Holomorphic disks and knot invariants. Adv. Math., 186(1):58116, 2004.

[OS05] Peter Ozsváth and Zoltán Szabó. On the Heegaard Floer homology of branched double-covers. Adv. Math., 194(1):1-33, 2005.

[OSS17] Peter S. Ozsváth, András I. Stipsicz, and Zoltán Szabó. Concordance homomorphisms from knot Floer homology. Adv. Math., 315:366-426, 2017.

[Pla06] Olga Plamenevskaya. Transverse knots and Khovanov homology. Math. Res. Lett., 13(4):571-586, 2006.

[Ras03] Jacob Andrew Rasmussen. Floer homology and knot complements. ProQuest LLC, Ann Arbor, MI, 2003. Thesis (Ph.D.)-Harvard University.

[Ras10] Jacob Rasmussen. Khovanov homology and the slice genus. Inventiones mathematicae, 182(2):419-447, 2010.

[Rob13] Lawrence P. Roberts. On knot Floer homology in double branched covers. Geom. Topol., 17(1):413-467, 2013.

[Rud83] Lee Rudolph. Braided surfaces and Seifert ribbons for closed braids. Comment. Math. Helv., 58(1):1-37, 1983.

[Sal17] Adam Saltz. Strong Khovanov-Floer theories and functoriality, 2017. preprint, arXiv:1712.08272.

[Shu07] Alexander N. Shumakovitch. Rasmussen invariant, slice-Bennequin inequality, and sliceness of knots. J. Knot Theory Ramifications, 16(10):1403-1412, 2007.

[SSS17] Sucharit Sarkar, Cotton Seed, and Zoltán Szabó. A perturbation of the geometric spectral sequence in Khovanov homology. Quantum Topol., 8(3):571-628, 2017.

[Sza15] Zoltán Szabó. A geometric spectral sequence in khovanov homology. Journal of Topology, 8(4):1017-1044, 2015.

[Tur06] Paul R. Turner. Calculating Bar-Natan's characteristic two Khovanov homology. J. Knot Theory Ramifications, 15(10):1335-1356, 2006.

School of Mathematics, Institute for Advanced Study, Princeton, NJ 08540

E-mail address: ltruong@math.ias.edu

Department of Mathematics, University of Georgia, Athens, GA 30602

E-mail address: melissa.zhang@uga.edu 